\title{
Synthesis of $\alpha$-Fluorosulfonamides by Electrophilic Fluorination
}

\author{
Bryan Hill, Yong Liu and Scott D. Taylor \\ Department of Chemistry, University of Waterloo, 200 University Avenue West, Waterloo, Ontario, \\ Canada, N2L $3 G 1$.
}

\section{Supporting Information}

General. All starting materials and reagents were obtained from Aldrich Chemical Company (Oakville, ON, Canada. NSFI was purchased from Synquest and recrystallized from hot methanol prior to use. Tetrahydrofuran (THF) was distilled from sodium metal in the presence of benzophenone under argon. $\mathrm{CH}_{2} \mathrm{Cl}_{2}$ was distilled from calcium hydride under nitrogen. Both DMF and HMPA were distilled under reduced pressure from calcium hydride and stored over 4 angstrom sieves under argon. Silica gel chromatography was performed using silica gel 60A (230-400 mesh) obtained from Silicycle (Laval, Quebec, Canada). ${ }^{1} \mathrm{H},{ }^{19} \mathrm{~F}$, and ${ }^{13} \mathrm{C}$ NMR spectra were recorded on a Bruker Avance 300 spectrometer. Chemical shifts $(\delta)$ for ${ }^{1} \mathrm{H}$ NMR spectra run in $\mathrm{CDCl}_{3}$ are reported in $\mathrm{ppm}$ relative to the internal standard tetramethylsilane (TMS). For acetone- $\mathrm{d}_{6}(\delta=2.04)$, DMSO-d $\mathrm{d}_{6}(\delta=2.49)$. For ${ }^{13} \mathrm{C} \mathrm{NMR}$ spectra run in $\mathrm{CDCl}_{3}$, chemical shifts are reported in ppm relative to the $\mathrm{CDCl}_{3}(\delta=77.0$ for central peak $)$, acetone- $\mathrm{d}_{6}(\delta$ $=29.8$ for central peak $)$, DMSO $(\delta=39.5$ for central peak $)$. For the ${ }^{19} \mathrm{~F} \mathrm{NMR}$ spectra chemicals shifts are reported relative to an external fluoroform standard $\left(\delta=0\right.$ for $\left.\mathrm{CFCl}_{3}\right)$. Melting points were determined on a Fisher-Johns melting point apparatus and are uncorrected.

General Procedure for the synthesis of Sulfonamides (7 to 9, 15 to 24)

To a stirred $0^{\circ} \mathrm{C}$ solution of secondary amine 4, 5, or $\mathbf{6}$ (1.1 eq), DMAP (2.0 eq) and THF $(0.3 \mathrm{M})$ was added a solution of sulfonyl chlorides (1.0eq) and THF. The ice bath was removed and the resulting mixture stirred overnight at room temperature. The mixture was filtered, washed with EtOAc. The filtrate was washed with aq $\mathrm{NH}_{4} \mathrm{Cl}$, brine, dried over $\mathrm{MgSO}_{4}$ and concentrated. Purification by column chromatography (15:85 to 25:75 EtOAc:Hexanes) yielded pure sulfonamides.<smiles>O=S(=O)(Cc1ccccc1)N(Cc1ccccc1)Cc1ccccc1</smiles> 
$N, N$-Dibenzyl-1-phenylmethanesulfonamide (7): Benzylsulfonyl chloride (1.00 g, 5.24 mmole), dibenzylamine (1.24 g, $6.29 \mathrm{mmole})$, triethylamine $(637 \mathrm{mg}, 6.29 \mathrm{mmole})$ in THF (15 mL). Flash chromatography (10:90 to 15:85 EtOAc:Hexanes) yielded $1.58 \mathrm{~g}$ $(86 \%)$ as a white crystalline solid. M.p. $98-99^{\circ} \mathrm{C} ;{ }^{1} \mathrm{H}$ NMR (300 MHz): $\delta$ 7.37-7.24 (13H, $\mathrm{m}), 7.03-6.99(2 \mathrm{H}, \mathrm{m}), 4.13(2 \mathrm{H}, \mathrm{s}), 4.09(4 \mathrm{H}, \mathrm{s}) ;{ }^{13} \mathrm{C} \mathrm{NMR}(75 \mathrm{MHz}): \delta 135.5,130.4$, 128.6, 128.3, 128.2, 127.6, 59.3, 50.3; LRMS (EI) $m z$ (relative intensity): $351\left(\mathrm{M}^{+}, 2\right)$, $260\left(\mathrm{M}^{+}-\mathrm{C}_{7} \mathrm{H}_{7}, 30\right), 91(100) ;\left(\mathrm{CI}, \mathrm{NH}_{3}\right): 369\left(\mathrm{M}^{+}+\mathrm{NH}_{4}, 100\right), 352\left(\mathrm{M}^{+}+\mathrm{H}, 20\right)$; HRMS (EI) $m / z$ calculated for $\mathrm{C}_{21} \mathrm{H}_{21} \mathrm{NO}_{2} \mathrm{~S}: 351.1293$ found 351.1310

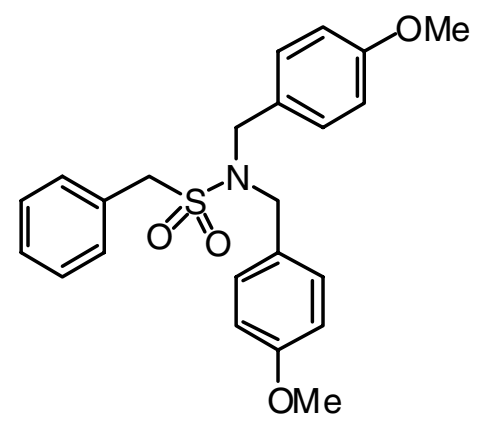

$\mathrm{N}, \mathrm{N}$-Bis(4-methoxybenzyl)-1-phenylmethanesulfonamide (8): Benzylsulfonyl chloride (1.23 g, $6.45 \mathrm{mmole})$, amine 5 (3.32 g, $12.9 \mathrm{mmole})$ in THF (14 mL). Flash chromatography (15:85 to 40:60 EtOAc:Hexanes) yielded $1.86 \mathrm{~g}(70 \%)$ as a white

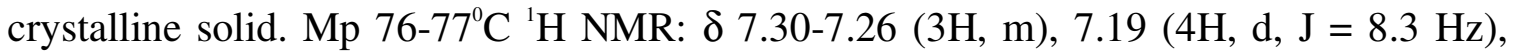
$7.05(2 \mathrm{H}, \mathrm{d}, \mathrm{J}=6.5 \mathrm{~Hz}), 6.87(4 \mathrm{H}, \mathrm{d}, \mathrm{J}=8.3 \mathrm{~Hz}), 4.10(2 \mathrm{H}, \mathrm{s}), 4.03(4 \mathrm{H}, \mathrm{s}), 3.81(6 \mathrm{H}, \mathrm{s})$; ${ }^{13}$ C NMR: $\delta$ 159.2, 130.6, 130.2, 128.9, 128.4, 128.4, 127.8, 113.8, 59.6, 55.1, 49.6; LRMS (EI) $m z$ (relative intensity): $411\left(\mathrm{M}^{+}, 9\right), 121$ (100), 91 (73); HRMS (EI) $m / z$ calculated for $\mathrm{C}_{23} \mathrm{H}_{25} \mathrm{NO}_{4} \mathrm{~S}: 411.150430$, found 411.151740 .<smiles>COc1ccc(CN(Cc2ccc(OC)cc2OC)S(=O)(=O)Cc2ccccc2)c(OC)c1</smiles>

$N, N$-Bis(2,4-dimethoxybenzyl)-1-phenylmethanesulfonamide (9): Benzylsulfonyl chloride (1.00 g, 5.24 mmole), amine 6 (1.83 g, 5.77 mmole), DMAP (705 mg, 5.77 mmole) in THF ( $15 \mathrm{~mL}$ ). Flash chromatography (25:75 EtOAc:Hexanes) yielded $1.935 \mathrm{~g}$ $(78 \%)$ as a white crystalline solid. Mp $116-117^{\circ} \mathrm{C} .{ }^{1} \mathrm{H}$ NMR: $\delta$ 7.30-7.24 $(5 \mathrm{H}, \mathrm{m}), 7.14-$ $7.11(2 \mathrm{H}, \mathrm{m}), 6.48-6.44(4 \mathrm{H}, \mathrm{m}), 4.18(4 \mathrm{H}, \mathrm{s}), 4.06(2 \mathrm{H}, \mathrm{s}), 3.81(6 \mathrm{H}, \mathrm{s}), 3.78(6 \mathrm{H}, \mathrm{s}),{ }^{13} \mathrm{C}$ NMR: $\delta$ 160.3, 158.2, 131.1, 130.6, 129.3, 128.3, 128.1, 117.1, 103.8, 98.1, 58.9, 55.2, 
54.9, 45.3; LRMS (EI) $m / z$ (relative intensity): $471\left(\mathrm{M}^{+}, 15\right), 151$ (100), 178 (45); HRMS (EI) $m / z$ calculated for $\mathrm{C}_{25} \mathrm{H}_{29} \mathrm{NO}_{6} \mathrm{~S}: 471.171560$, found 471.171604 .<smiles>COc1ccc(CN(Cc2ccc(OC)cc2OC)S(=O)(=O)Cc2ccc(Br)cc2)c(OC)c1</smiles>

$\mathrm{N}, \mathrm{N}$-Bis(2,4-dimethoxybenzyl)-1-(4-bromophenyl)methanesulfonamide $\quad$ (15): $\quad 4$ Bromobenzylsulfonyl chloride ${ }^{1}(1.93 \mathrm{~g}, 7.16 \mathrm{mmole})$, amine 6 (2.72 g, $\left.8.59 \mathrm{mmole}\right)$, DMAP $(1.05 \mathrm{~g}, 8.59 \mathrm{mmole})$ in THF $(70 \mathrm{~mL})$. Flash chromatography $(25: 75$ EtOAc:Hexanes) yielded $2.85 \mathrm{~g}(72 \%)$ as a white crystalline solid. $\mathrm{Mp} 119-121^{\circ} \mathrm{C} .{ }^{1} \mathrm{H}$ NMR: $\delta 7.38(2 \mathrm{H}, \mathrm{d}, \mathrm{J}=8.3 \mathrm{~Hz}), 7.23(2 \mathrm{H}, \mathrm{d}, \mathrm{J}=8.6 \mathrm{~Hz}), 6.97(2 \mathrm{H}, \mathrm{d}, \mathrm{J}=8.3 \mathrm{~Hz}), 6.47-$ $6.44(4 \mathrm{H}, \mathrm{m}), 4.20(4 \mathrm{H}, \mathrm{s}), 3.99(2 \mathrm{H}, \mathrm{s}), 3.78(6 \mathrm{H}, \mathrm{s}), 3.77(6 \mathrm{H}, \mathrm{s}) ;{ }^{13} \mathrm{C}$ NMR: $\delta 160.4$, 158.2, 132.2, 131.4, 131.1, 128.5, 122.3, 116.9, 103.9, 98.1, 58.2, 55.2, 55.0, 45.3; LRMS (EI) $m z$ (relative intensity): $549\left(\mathrm{M}^{+}, 14\right), 551\left(\mathrm{M}^{+}, 15\right), 151$ (100), 178 (64); HRMS (EI) $m / z$ calculated for $\mathrm{C}_{25} \mathrm{H}_{28} \mathrm{BrNO}_{6} \mathrm{~S}$ : 549.082071, found 549.081497.<smiles>COc1ccc(CN(Cc2ccc(OC)cc2OC)S(=O)(=O)Cc2ccc(I)cc2)c(OC)c1</smiles>

$\boldsymbol{N}, \boldsymbol{N}$-Bis(2,4-dimethoxybenzyl)-1-(4-iodophenyl)methanesulfonamide $\quad$ (16): 4Iodobenzylsulfonyl chloride $^{1}$ (9.4 g, 29.7 mmole), amine 6 (12.5 g, 39.4 mmole), DMAP (360 mg, $2.97 \mathrm{mmole})$, triethylamine $(7.69 \mathrm{~g}, 76.0 \mathrm{mmole})$ in THF (100 mL). Flash chromatography (25:75 to 33:67 EtOAc:Hexanes) yielded $10.4 \mathrm{~g}$ (59 \%) as a white crystalline solid. Mp $130^{\circ} \mathrm{C} .{ }^{1} \mathrm{H}$ NMR: $\delta 7.60(2 \mathrm{H}, \mathrm{d}, \mathrm{J}=8.2 \mathrm{~Hz}), 7.24(2 \mathrm{H}, \mathrm{d}, \mathrm{J}=8.2 \mathrm{~Hz})$, $6.84(2 \mathrm{H}, \mathrm{d}, \mathrm{J}=8.1 \mathrm{~Hz}), 6.47-6.45$ (4H, m), 4.19 (4H, s), 3.97 (2H, s), $3.81(6 \mathrm{H}, \mathrm{s}), 3.79$ $(6 \mathrm{H}, \mathrm{s}) ;{ }^{13} \mathrm{C} \mathrm{NMR}: \delta 160.5,158.3,137.5,132.5,131.3,129.2,117.1,104.0,98.3,94.2$, 58.6, 55.4, 55.1, 45.4; LRMS (EI) $m / z$ (relative intensity): $620\left(\mathrm{M}^{+}+\mathrm{Na}, 100\right), 151$ (71); HRMS (EI) $m z$ calculated for $\mathrm{C}_{25} \mathrm{H}_{28} \mathrm{NO}_{6} \mathrm{SINa}$ : 620.0574, found 620.0554 
<smiles>COc1ccc(CN(Cc2ccc(OC)cc2OC)S(=O)(=O)Cc2ccc([N+](=O)[O-])cc2)c(OC)c1</smiles>

$\mathrm{N}, \mathrm{N}$-Bis(2,4-dimethoxybenzyl)-1-(4-nitrophenyl)methanesulfonamide (17): 4Nitrobenzylsulfonyl chloride ${ }^{1}$ ( $2.0 \mathrm{~g}, 8.48$ mmole), amine 6 (3.17 g, 10.0 mmole), DMAP $(1.24 \mathrm{~g}, 10.2 \mathrm{mmole})$ in THF $(80 \mathrm{~mL})$. Flash chromatography (25:75 EtOAc:Hexanes) yielded $1.91 \mathrm{~g}(44 \%)$ as a slightly yellow crystalline solid. Mp 136-137 ${ }^{\circ} \mathrm{C} .{ }^{1} \mathrm{H}$ NMR: $\delta$ $8.10(2 \mathrm{H}, \mathrm{d}, \mathrm{J}=8.4 \mathrm{~Hz}), 7.28(2 \mathrm{H}, \mathrm{d}, \mathrm{J}=8.5 \mathrm{~Hz}), 7.24(2 \mathrm{H}, \mathrm{d}, \mathrm{J}=9.0 \mathrm{~Hz}), 6.47-6.46(4 \mathrm{H}$, $\mathrm{m}), 4.23(4 \mathrm{H}, \mathrm{s}), 4.13(2 \mathrm{H}, \mathrm{s}), 3.80(12 \mathrm{H}, \mathrm{s}) ;{ }^{13} \mathrm{C}$ NMR: $\delta 160.5,158.1,147.4,136.8$, 131.5, 131.1, 123.3, 116.5, 104.0, 98.1, 58.2, 55.2, 55.0, 45.3; LRMS (EI) $m z$ (relative intensity): $516\left(\mathrm{M}^{+}, 9\right), 151$ (100), 178 (84); HRMS (EI) $m / z$ calculated for $\mathrm{C}_{25} \mathrm{H}_{28} \mathrm{~N}_{2} \mathrm{O}_{8} \mathrm{~S}$ : 516.156638 , found 516.156073 .<smiles>COc1ccc(CN(Cc2ccc(OC)cc2OC)S(=O)(=O)Cc2ccc(C)cc2)c(OC)c1</smiles>

$\mathrm{N}, \mathrm{N}$-Bis(2,4-dimethoxybenzyl)-1-(4-methylphenyl)methanesulfonamide $\quad(18): \quad 4-$ Methylbenzylsulfonyl chloride ${ }^{1}$ (763 mg, 3.72 mmole), amine 6 (478 mg, $3.91 \mathrm{mmole}$ ), DMAP (478 mg, 3.91 mmole) in THF $(40 \mathrm{~mL})$. Flash chromatography $(25: 75$ EtOAc:Hexanes) yielded $837 \mathrm{mg}(46 \%)$ as a white crystalline solid. Mp $82-84^{\circ} \mathrm{C} .{ }^{1} \mathrm{H}$ NMR: $\delta 7.25(2 \mathrm{H}, \mathrm{d}, \mathrm{J}=7.3 \mathrm{~Hz}), 7.09(2 \mathrm{H}, \mathrm{d}, \mathrm{J}=8.0 \mathrm{~Hz}), 7.02(2 \mathrm{H}, \mathrm{d}, \mathrm{J}=8.0 \mathrm{~Hz}), 6.47$ $6.43(4 \mathrm{H}, \mathrm{m}), 4.19(4 \mathrm{H}, \mathrm{s}), 4.02(2 \mathrm{H}, \mathrm{s}), 3.80(6 \mathrm{H}, \mathrm{s}), 3.77(6 \mathrm{H}, \mathrm{s}), 2.32(3 \mathrm{H}, \mathrm{s}) ;{ }^{13} \mathrm{C}$ NMR: $\delta$ 160.3, 158.1, 137.8, 131.1, 130.4, 128.9, 126.3, 117.1, 103.9, 98.0, 58.5, 55.1, 54.9, 45.3, 20.9; LRMS (EI) $m / z$ (relative intensity): $485\left(\mathrm{M}^{+}, 10\right), 151$ (100), 178 (51); HRMS (EI) $m / z$ calculated for $\mathrm{C}_{26} \mathrm{H}_{31} \mathrm{NO}_{6} \mathrm{~S}: 485.187210$, found 485.187596 . 
<smiles>COc1ccc(CN(Cc2ccc(OC)cc2OC)S(=O)(=O)Cc2cccc(Br)c2)c(OC)c1</smiles>

$\mathrm{N}, \mathrm{N}$-Bis(2,4-dimethoxybenzyl)-1-(3-bromophenyl)methanesulfonamide $\quad$ (19): $\quad 3$ Bromobenzylsulfonyl chloride (475 mg, $1.76 \mathrm{mmole}$ ), amine 6 (617 mg, $1.94 \mathrm{mmole}$ ), DMAP (238 mg, $1.94 \mathrm{mmole})$ in THF $(15 \mathrm{~mL})$. Flash chromatography (25:75 EtOAc:Hexanes) yielded $723 \mathrm{mg}(75 \%)$ as a white crystalline solid. $\mathrm{Mp} 118-120^{\circ} \mathrm{C} .{ }^{1} \mathrm{H}$ NMR: $\delta$ 7.42-7.38 $(1 \mathrm{H}, \mathrm{m}), 7.26(2 \mathrm{H}, \mathrm{d}, \mathrm{J}=8.7 \mathrm{~Hz}), 7.13(2 \mathrm{H}, \mathrm{d}, \mathrm{J}=4.9 \mathrm{~Hz}), 7.06(1 \mathrm{H}$, s), 6.48-6.46 (4H, m), $4.20(4 \mathrm{H}, \mathrm{s}), 3.96(2 \mathrm{H}, \mathrm{s}), 3.79(12 \mathrm{H}, \mathrm{s}) ;{ }^{13} \mathrm{C}$ NMR: $\delta 160.4,158.2$, $133.4,131.6,131.1,129.8,129.3,122.0,116.9,104.0,98.2,58.5,55.2,55.0,45.4$;

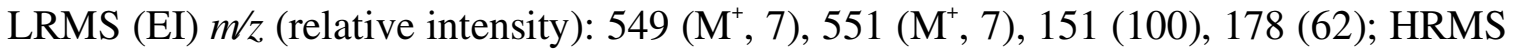
(EI) $m / z$ calculated for $\mathrm{C}_{25} \mathrm{H}_{28} \mathrm{BrNO}_{6} \mathrm{~S}$ : 549.082071, found 549.081480.
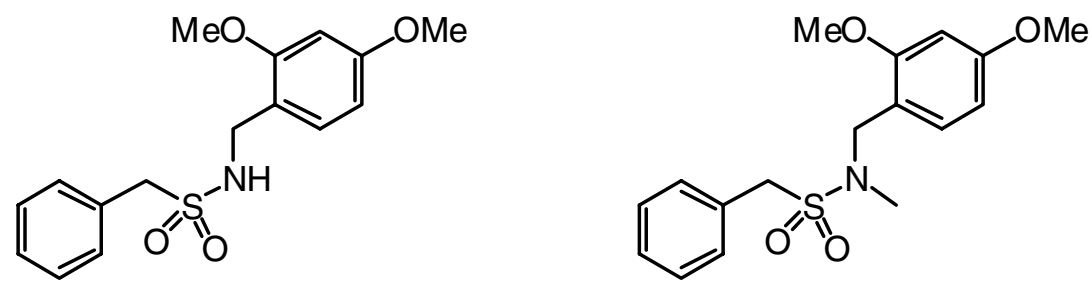

$N$-Methyl- $N$-(2,4-dimethoxybenzyl)-1-phenylmethanesulfonamide (20): A solution of benzylsulfonyl chloride ( $627 \mathrm{mg}, 3.29 \mathrm{mmole})$ and THF $(8 \mathrm{~mL}+2 \mathrm{~mL}$ rinse) was added via cannula to a $0^{\circ} \mathrm{C}$ solution of amine $6(250 \mathrm{mg}, 1.50 \mathrm{mmole}), i \mathrm{Pr}_{2} \mathrm{NEt}(650 \mu \mathrm{L}, 3.74$ mmole), and THF $(5 \mathrm{~mL})$. The ice bath was removed and the reaction stirred at room temperature for $4 \mathrm{hr}$. The mixture was filtered and washed with EtOAc, the filtrate washed with aq $\mathrm{NH}_{4} \mathrm{Cl}$, brine, dried over $\mathrm{MgSO}_{4}$, filtered and concentrated. Flash chromatography (25:75 to $33: 67$ to 40:60 EtOAc:Hexanes) yielded $467 \mathrm{mg}(98 \%)$ as a yellow solid. Mp 86-87 ${ }^{\circ} \mathrm{C} ;{ }^{1} \mathrm{H}$ NMR (300 MHz): $\delta$ 7.32-7.27 (3H, m), 7.17-7.10 (3H, m), 6.48-6.45 (2H, m), $4.74(1 \mathrm{H}, \mathrm{bm}), 4.18(2 \mathrm{H}, \mathrm{d}, \mathrm{J}=6.2 \mathrm{~Hz}), 4.08(2 \mathrm{H}, \mathrm{s}), 3.82(3 \mathrm{H}, \mathrm{s})$, $3.75(3 \mathrm{H}, \mathrm{s}) ;{ }^{13} \mathrm{C}$ NMR $(75 \mathrm{MHz}): \delta 160.9,158.4,130.4,130.4,129.2,128.4,128.2$, 117.6, 103.8, 98.5, 58.9, 55.3, 55.0, 43.4; LRMS (EI) $m z$ (relative intensity): $321\left(\mathrm{M}^{+}\right.$, 55), 166 (100), 91(80), 151 (58); HRMS (EI) $m / z$ calculated for $\mathrm{C}_{16} \mathrm{H}_{19} \mathrm{NO}_{4} \mathrm{~S}: 321.1035$ found: 321.1033. Part 2: To a stirred $0^{\circ} \mathrm{C}$ solution of intermediate sulfonamide $(351 \mathrm{mg}$, $1.09 \mathrm{mmole}), \mathrm{PPh}_{3}(571 \mathrm{mg}, 2.18 \mathrm{mmole}), \mathrm{MeOH}(88 \mu \mathrm{L}, 2.18 \mathrm{mmole})$ and THF (7.3 $\mathrm{mL}$ ) was added DIAD (441 mg, 2.18 mmole) dropwise. After 15 minutes the ice bath was removed and the reaction stirred overnight. The solvent was removed and the residue purified by flash chromatography (15:85 to 25:75 EtOAc:Hexanes, $2^{\text {nd }}$ column 
40:60 $\mathrm{Et}_{2} \mathrm{O}$ :Toluene) yielded $315 \mathrm{mg}(86 \%)$ of sulfonamide 20 as a white solid. Mp 37$40^{\circ} \mathrm{C} ;{ }^{1} \mathrm{H}$ NMR (300 MHz): $\delta$ 7.37-7.34 $(5 \mathrm{H}, \mathrm{m}), 7.23(1 \mathrm{H}, \mathrm{d}, \mathrm{J}=8.0 \mathrm{~Hz}), 6.48-6.46(2 \mathrm{H}$, $\mathrm{m}), 4.20(4 \mathrm{H}, \mathrm{d}, \mathrm{J}=4.2 \mathrm{~Hz}), 3.81(3 \mathrm{H}, \mathrm{s}), 3.80(3 \mathrm{H}, \mathrm{s}), 2.59(3 \mathrm{H}, \mathrm{s}) ;{ }^{13} \mathrm{C}$ NMR: $\delta 160.5$, 158.3, 130.9, 130.5, 129.1, 128.4, 128.3, 116.4, 104.2, 98.2, 57.0, 55.1, 55.0, 47.7, 34.9; LRMS (EI) $m z$ (relative intensity): $335\left(\mathrm{M}^{+}, 45\right), 151$ (100), 180 970); HRMS (EI) $m / z$ calculated for $\mathrm{C}_{17} \mathrm{H}_{21} \mathrm{NO}_{4} \mathrm{~S}: 335.1191$ found 335.1203.<smiles>COc1ccc(CN(c2ccccc2)S(=O)(=O)Cc2ccccc2)c(OC)c1</smiles>

$N$-Phenyl- $N$-(2,4-dimethoxybenzyl)-1-phenylmethanesulfonamide

(21):

Benzylsulfonyl chloride (184 mg, 0.967 mmole), N,N-phenyl-2,4-dimethoxylbenzyl amine $^{2}$ (351 mg, $\left.1.45 \mathrm{mmole}\right)$, DMAP (118 mg, $\left.0.967 \mathrm{mmole}\right)$ in THF (5mL). Flash chromatography (15:85 to $25: 75$ to $40: 60$ EtOAc:Hexanes) yielded $289 \mathrm{mg}(75 \%)$ as a white crystalline solid. Mp 143-144 ${ }^{\circ} \mathrm{C}{ }^{1} \mathrm{H}$ NMR: $\delta$ 7.39-7.38 $(5 \mathrm{H}, \mathrm{m}), 7.24-7.19(3 \mathrm{H}, \mathrm{m})$, 7.12-7.09 (3H, m), 6.38-6.31 (2H, m), $4.66(2 \mathrm{H}, \mathrm{s}), 4.30(2 \mathrm{H}, \mathrm{s}), 3.75(3 \mathrm{H}, \mathrm{s}), 3.60(3 \mathrm{H}$, s); ${ }^{13} \mathrm{C}$ NMR: $\delta$ 160.4, 158.2, 139.5, 131.2, 130.8, 128.9, 128.8, 128.7, 128.6, 128.5, 127.2, 116.9, 104.0, 98.1, 57.6, 55.1, 55.0, 49.5; LRMS (EI) $m / z$ (relative intensity): 397 $\left(\mathrm{M}^{+}, 2\right), 151$ (100); HRMS (EI) $m z$ calculated for $\mathrm{C}_{22} \mathrm{H}_{23} \mathrm{NO}_{4} \mathrm{~S}$ : 397.134780, found 397.134694 .<smiles>CN(C)S(=O)(=O)Cc1ccccc1</smiles>

$N, N$-Dimethyl-1-phenylmethanesulfonamide (22): Prepared with slight modifications to known literature procedures ${ }^{3}$. Benzylsulfonyl chloride (2.68 g, 14.0 mmole), [2.0M] dimethylamine ( $15 \mathrm{~mL}, 30.0$ mmole), THF $(15 \mathrm{~mL})$. Recrystallization from hot ethanol yielded $1.92 \mathrm{~g}(69 \%)$ as a white flakey crystalline solid. $\mathrm{Mp} 98^{\circ} \mathrm{C}\left(\text { lit } 99-105^{\circ} \mathrm{C}\right)^{3}{ }^{1} \mathrm{H}$ NMR: $\delta$ 7.39-7.35 (5H, m), $4.23(2 \mathrm{H}, \mathrm{s}), 2.72(6 \mathrm{H}, \mathrm{s}) ;{ }^{13} \mathrm{C}$ NMR: $\delta 130.4,128.8,128.5$, $128.4,55.3,37.4$<smiles>CN([13CH3])S(=O)(=O)Cc1ccccc1</smiles> 
$N$-Methyl- $N$-(dimethyl-tert-butylsilyl)-1-phenylmethanesulfonamide (23): To a $0^{0} \mathrm{C}$ suspension of oil free KH (107 mg, 2.69 mmole) and THF $(10 \mathrm{~mL})$ was added a solution of sulfonamide 43 (355mg, 1.92 mmole), 18-C-6 (253 mg, 0.96 mmole) and THF (5 mL $+4 \mathrm{~mL}$ rinse) via cannula. The reaction was stirred 2 hours further at $0^{\circ} \mathrm{C}$ before TBSCl (434 mg, 2.88 mmole) was added. After 1 hour at $0^{\circ} \mathrm{C}$, the reaction was quenched with aq $\mathrm{NH}_{4} \mathrm{Cl}$. The product was extracted with EtOAc (x3), washed with aq $\mathrm{NaHCO}_{3}$, brine, dried over $\mathrm{MgSO}_{4}$, filtered and concentrated. Flash chromatography (15:85 to 25:75 to 40:60 EtOAc:Hexanes) yielded $490 \mathrm{mg}(85 \%)$ of sulfonamide 23 as a white solid. M.p. $89-90^{\circ} \mathrm{C} ;{ }^{1} \mathrm{H}$ NMR $(300 \mathrm{MHz}): \delta 7.37-7.34(5 \mathrm{H}, \mathrm{m}), 4.19(2 \mathrm{H}, \mathrm{s}), 2.78(3 \mathrm{H}, \mathrm{s}), 0.91(9 \mathrm{H}$, s), -0.08 (6H, s); ${ }^{13} \mathrm{C}$ NMR (75 MHz): $\delta 130.8,129.8,128.6,128.5,56.8,34.3,26.6,19.6$, -4.3; LRMS (EI) $m z$ (relative intensity): $242\left(\mathrm{M}^{+}-\mathrm{C}_{4} \mathrm{H}_{9}, 28\right), 91(100), 165(48)$; HRMS (EI) $m / z$ calculated for $\mathrm{C}_{10} \mathrm{H}_{16} \mathrm{NO}_{2} \mathrm{SSi}\left(\mathrm{M}^{+}-\mathrm{C}_{4} \mathrm{H}_{9}\right): 242.067104$ found 242.067146.

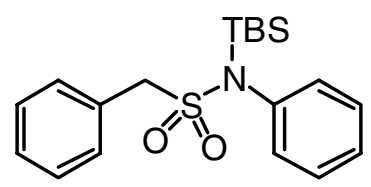

$\boldsymbol{N}$-Phenyl- $\boldsymbol{N}$-(dimethyl-tert-butylsilyl)-1-phenylmethanesulfonamide (24): To a stirred $0^{\circ} \mathrm{C}$ solution of sulfonamide $42(408 \mathrm{mg}, 1.65 \mathrm{mmole})$ and THF (16.5 mL) was added $\mathrm{NaH}\left(60 \%\right.$ dispersion) $(86 \mathrm{mg}, 2.14 \mathrm{mmole})$. After 1 hour at $0^{\circ} \mathrm{C} \mathrm{TBSCl}(373 \mathrm{mg}, 2.48$ mmole) was added and the ice bath was removed. A thick white solid formed. DMF (10 $\mathrm{mL}$ ) was added to solublize the solid and the mixture was stirred overnight. The reaction was quenched with $\mathrm{H}_{2} \mathrm{O}$, extracted with $\mathrm{Et}_{2} \mathrm{O}(\mathrm{x} 3)$, washed with $\mathrm{H}_{2} \mathrm{O}(\mathrm{x} 2)$, brine, dried over $\mathrm{MgSO}_{4}$, filtered and concentrated to dryness. Flash chromatography (5:95 to 15:85 EtOAc: Hexanes) yielded $231 \mathrm{mg}$ (39\%) of sulfonamide $\mathbf{2 4}$ as a white crystalline solid while recovering $222 \mathrm{mg}$ (54\%) of sulfonamide 42. M.p. $67-70{ }^{\circ} \mathrm{C} ;{ }^{1} \mathrm{H}$ NMR $(300 \mathrm{MHz})$ : $\delta$ 7.36-7.34 (10H, m), 4.15 (2H, s), $0.97(9 \mathrm{H}, \mathrm{s}), 0.00(6 \mathrm{H}, \mathrm{s}) ;{ }^{13} \mathrm{C}$ NMR $(75 \mathrm{MHz}): \delta 139.0$, 131.6, 131.0,129.0, 128.9, 128.6, 128.5, 127.9, 57.2, 27.5, 19.9, -2.6; LRMS (EI) $m / z$ (relative intensity): $361\left(\mathrm{M}^{+}, 25\right), 304\left(\mathrm{M}^{+}-\mathrm{C}_{4} \mathrm{H}_{9}, 17\right), 91$ (100), 240 (58); HRMS (EI) $\mathrm{m} z$ calculated for $\mathrm{C}_{19} \mathrm{H}_{27} \mathrm{NO}_{2} \mathrm{SSi}$ : 361.153180, found 361.153708 .

\section{General Procedure for the fluorination of Benzylic Sulfonamides 10 to 12 and 25 to 34.}

To a cooled $-78^{\circ} \mathrm{C}$ solution of sulfonamide (1.00 eq), NSFI (2.5 eq), and THF (0.025M) was added [1.0 M] NaHMDS in THF (2.2 eq) dropwise over $0.5 \mathrm{hr}$ via syringe pump. The reaction was stirred at $-78^{\circ} \mathrm{C}$ for $2 \mathrm{hrs}$ further then warmed to $\mathrm{rt}$ and stirred another 2 hrs. The reaction was quenched with aq $\mathrm{NH}_{4} \mathrm{Cl}$, and separated. The aqueous was extracted with EtOAc (x3), and the combined organics were washed with aq $\mathrm{NaHCO}_{3}$, brine, dried $\left(\mathrm{MgSO}_{4}\right)$, filtered, and concentrated. Purification by flash chromatography (15:85 to 25:75 EtOAc: Hexanes) yielded pure sulfonamides that could be recrystallized from EtOAc:Hexanes. 
<smiles>O=S(=O)(N(Cc1ccccc1)Cc1ccccc1)C(F)(F)c1ccccc1</smiles>

$N, N$-Dibenzyl-1,1-difluoro-1-phenylmethanesulfonamide (10): Sulfonamide 7 (187 mg, $0.532 \mathrm{mmole}$ ), NSFI (419 mg, $1.33 \mathrm{mmole})$, NaHMDS (1.2 mL, $1.20 \mathrm{mmole}$ ), in THF (21 mL). Flash chromatography (5:95 EtOAc:Hexanes) yielded $156 \mathrm{mg}(76 \%)$ as a off-white, pale yellow crystalline solid. Mp 92-93 ${ }^{\circ} \mathrm{C} ;{ }^{1} \mathrm{H}$ NMR $(300 \mathrm{MHz}): \delta 7.70(2 \mathrm{H}, \mathrm{d}$, $\mathrm{J}=7.4 \mathrm{~Hz}), 7.56-7.46(3 \mathrm{H}, \mathrm{m}), 7.31-7.21(10 \mathrm{H}, \mathrm{m}), 4.42(4 \mathrm{H}, \mathrm{s}) ;{ }^{13} \mathrm{C} \mathrm{NMR}(75 \mathrm{MHz}): \delta$ 134.7, 131.8, $128.9(\mathrm{t}, \mathrm{J}=22.6 \mathrm{~Hz}), 128.7,128.5,128.4,127.9,127.1(\mathrm{t}, \mathrm{J}=6.1 \mathrm{~Hz})$, $122.2\left(\mathrm{t}, \mathrm{J}=282.2 \mathrm{~Hz}\right.$ ), 51.2; ${ }^{19} \mathrm{~F}$ NMR (282 MHz): $\delta$-100.3; LRMS (EI) $m z$ (relative intensity): $387\left(\mathrm{M}^{+}, 1\right), 127\left(\mathrm{C}_{7} \mathrm{H}_{5} \mathrm{~F}_{2}, 100\right)$; HRMS (EI) $\mathrm{m} / z$ calculated for $\mathrm{C}_{21} \mathrm{H}_{19} \mathrm{~F}_{2} \mathrm{NO}_{2} \mathrm{~S}$ : 387.1105 found 387.1089<smiles>COc1ccc(CN(Cc2ccc(OC)cc2)S(=O)(=O)C(F)(F)c2ccccc2)cc1</smiles>

$\boldsymbol{N}, \boldsymbol{N}$-Bis(4-methoxybenzyl)-1,1-difluoro-1-phenylmethanesulfonamide (11): Sulfonamide 8 (400 mg, 0.973 mmole), NSFI (767 mg, 2.43 mmole), NaHMDS (2.2 mL, $2.2 \mathrm{mmole})$ and THF (39 mL). Flash chromatography (15:85 EtOAc:Hexanes) yielded $315 \mathrm{mg}(72 \%)$ as a white crystalline solid. $\mathrm{Mp} 85-86{ }^{\circ} \mathrm{C} ;{ }^{1} \mathrm{H}$ NMR $(300 \mathrm{MHz}): \delta 7.69$ $(2 \mathrm{H}, \mathrm{d}, \mathrm{J}=7.3 \mathrm{~Hz}), 7.54-7.47(3 \mathrm{H}, \mathrm{m}), 7.15(4 \mathrm{H}, \mathrm{d}, \mathrm{J}=8.6 \mathrm{~Hz}), 6.84(4 \mathrm{H}, \mathrm{d}, \mathrm{J}=8.6 \mathrm{~Hz})$, $4.33(4 \mathrm{H}, \mathrm{s}), 3.79(6 \mathrm{H}, \mathrm{s}) ;{ }^{13} \mathrm{C}$ NMR $(75 \mathrm{MHz}): \delta 159.3,131.8,130.2,129.0(\mathrm{t}, \mathrm{J}=22.8$ $\mathrm{Hz}), 128.5,127.2(\mathrm{t}, \mathrm{J}=6.0 \mathrm{~Hz}), 126.8,122.1(\mathrm{t}, \mathrm{J}=282.0 \mathrm{~Hz}), 113.9,55.2,50.3 ;{ }^{19} \mathrm{~F}$ NMR (282 MHz): $\delta$-100.4; LRMS (EI) $m z$ (relative intensity): $447\left(\mathrm{M}^{+}, 25\right), 121$ (100); HRMS (EI) $m z$ calculated for $\mathrm{C}_{23} \mathrm{H}_{23} \mathrm{~F}_{2} \mathrm{NO}_{4} \mathrm{~S}: 447.1316$, found 447.1333 


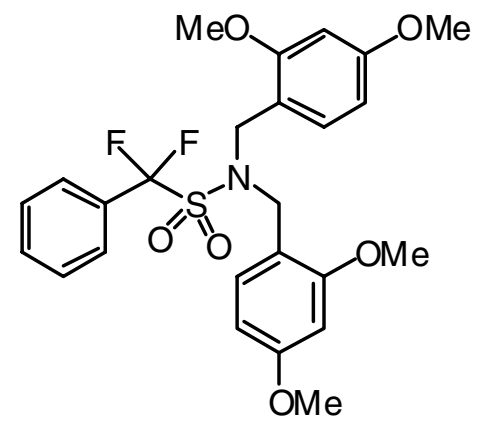

$N, N$-Bis(2,4-dimethoxybenzyl)-1,1-difluoro-1-phenylmethanesulfonamide

(12): Sulfonamide 9 (308mg, 0.653 mmole), NSFI (515mg, $1.63 \mathrm{mmole}$ ), NaHMDS (1.45 mL, $1.45 \mathrm{mmole})$ in THF $(26 \mathrm{~mL})$. Flash chromatography (25:75 EtOAc: Hexanes) yielded $269 \mathrm{mg}(81 \%)$ as a white crystalline solid. $\mathrm{Mp} 96-97^{\circ} \mathrm{C}{ }^{1} \mathrm{H}$ NMR $(300 \mathrm{MHz}): \delta 7.67$ $(2 \mathrm{H}, \mathrm{d}, \mathrm{J}=7.3 \mathrm{~Hz}), 7.54-7.44(3 \mathrm{H}, \mathrm{m}), 7.18(2 \mathrm{H}, \mathrm{d}, \mathrm{J}=8.4 \mathrm{~Hz}), 6.37(2 \mathrm{H}, \mathrm{dd}, \mathrm{J}=8.4,2.2$ $\mathrm{Hz}), 6.27(2 \mathrm{H}, \mathrm{d}, \mathrm{J}=2.1 \mathrm{~Hz}), 4.48(4 \mathrm{H}, \mathrm{s}), 3.77(6 \mathrm{H}, \mathrm{s}), 3.65(6 \mathrm{H}, \mathrm{s}) ;{ }^{13} \mathrm{C}$ NMR $(75$ MHz): $\delta 160.2,158.0,131.6,130.2,129.3(\mathrm{t}, \mathrm{J}=22.7 \mathrm{~Hz}), 128.3,127.0(\mathrm{t}, \mathrm{J}=6.0 \mathrm{~Hz})$, $122.2(\mathrm{t}, \mathrm{J}=282.6 \mathrm{~Hz}), 116.4,103.7,97.5,55.2,54.8,46.6 ;{ }^{19} \mathrm{~F}$ NMR $(282 \mathrm{MHz}): \delta$ 100.7; LRMS (EI) $m z$ (relative intensity): 507 ( $\left.\mathrm{M}^{+}, 43\right), 151$ (100), 178 (75), 127 (58); HRMS (EI) $m z$ calculated for $\mathrm{C}_{25} \mathrm{H}_{27} \mathrm{~F}_{2} \mathrm{NO}_{6} \mathrm{~S}: 507.152716$, found 507.152685.<smiles>COc1ccc(CN(Cc2ccc(OC)cc2OC)S(=O)(=O)C(F)(F)c2ccccc2)c(OC)c1</smiles>

$\mathrm{N}, \mathrm{N}$-Bis(2,4-dimethoxybenzyl)-1-fluoro-1-phenylmethanesulfonamide (14): Same as the general procedure. LDA was formed from $[1.15 \mathrm{M}] n$-BuLi $(370 \mu \mathrm{L}, 0.422 \mathrm{mmole})$ and $\mathrm{iPr}_{2} \mathrm{NH}(65 \mu \mathrm{L}, 0.464$ mmole $)$ in THF $(1 \mathrm{~mL})$ at -78 to $0^{\circ} \mathrm{C}$. Sulfonamide 9 (90.7 $\mathrm{mg}, 0.192 \mathrm{mmole})$, NSFI (152 mg, $0.481 \mathrm{mmole})$ and THF $(7.5 \mathrm{~mL})$. Flash chromatography (Benzene) yielded $55.5 \mathrm{mg}(59 \%)$ of sulfonamide 14 as a clear colorless film and $8.3 \mathrm{mg}$ of sulfonamide 12 ; ${ }^{1} \mathrm{H}$ NMR $(300 \mathrm{MHz}): \delta 7.40-7.38(5 \mathrm{H}, \mathrm{m}), 7.25(2 \mathrm{H}$, $\mathrm{d}, \mathrm{J}=8.0 \mathrm{~Hz}), 6.48-6.43(4 \mathrm{H}, \mathrm{m}), 5.66(1 \mathrm{H}, \mathrm{d}, \mathrm{J}=45.8 \mathrm{~Hz}), 4.47(2 \mathrm{H}, \mathrm{d}, \mathrm{J}=15.5 \mathrm{~Hz})$, $4.31(2 \mathrm{H}, \mathrm{d}, \mathrm{J}=15.5 \mathrm{~Hz}), 3.80(6 \mathrm{H}, \mathrm{s}), 3.78(6 \mathrm{H}, \mathrm{s}) ; 13 \mathrm{C} \mathrm{NMR}(75 \mathrm{MHz}): \delta 160.6$, 158.4, 131.1, $130.4(\mathrm{~d}, \mathrm{~J}=19.7 \mathrm{~Hz}), 130.1,128.3,127.6(\mathrm{~d}, \mathrm{~J}=6.3 \mathrm{~Hz}), 116.9,104.0$, $101.4(\mathrm{~d}, \mathrm{~J}=214.0 \mathrm{~Hz}), 98.3,55.3,55.1,46.1 ;{ }^{19} \mathrm{~F}$ NMR $(282 \mathrm{MHz}): \delta-173.67(\mathrm{~d}, \mathrm{~J}=$ $45.8 \mathrm{~Hz}$ ); LRMS (EI) $m / z$ (relative intensity): 489 (M+, 6), 151 (100), 178 (48); HRMS (EI) $m / z$ calculated for $\mathrm{C}_{25} \mathrm{H}_{28} \mathrm{FNO}_{6} \mathrm{~S}: 489.162138$, found 489.162897 . 


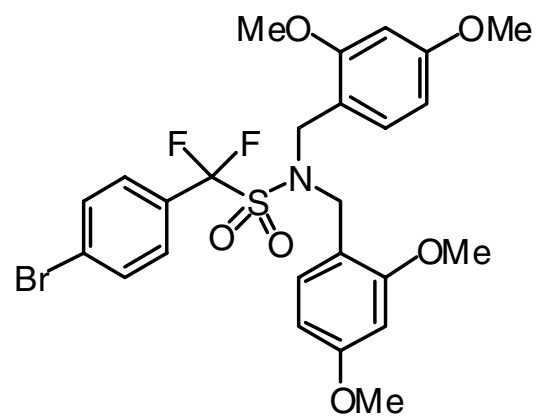

$\mathrm{N}, \mathrm{N}$-Bis(2,4-dimethoxybenzyl)-1,1-difluoro-1-(4-bromophenyl)methanesulfonamide (25): Sulfonamide 15 (228.8 mg, 0.416 mmole), NSFI (327 mg, 1.04 mmole), NaHMDS $(920 \mu \mathrm{L}, 0.920 \mathrm{mmole})$, and THF $(16 \mathrm{~mL})$. Flash chromatography (15:85 EtOAc: Hexanes) yielded $206 \mathrm{mg}(84 \%)$ as a clear crystalline solid. Mp $132-133^{\circ} \mathrm{C} ;{ }^{1} \mathrm{H}$ NMR $(300 \mathrm{MHz}): \delta 7.61(2 \mathrm{H}, \mathrm{d}, \mathrm{J}=8.5 \mathrm{~Hz}), 7.52(2 \mathrm{H}, \mathrm{d}, \mathrm{J}=8.5 \mathrm{~Hz}), 7.17(2 \mathrm{H}, \mathrm{d}, \mathrm{J}=8.4 \mathrm{~Hz})$, $6.38(2 \mathrm{H}, \mathrm{dd}, \mathrm{J}=8.4,2.3 \mathrm{~Hz}), 6.28(2 \mathrm{H}, \mathrm{d}, \mathrm{J}=2.3 \mathrm{~Hz}), 4.49(4 \mathrm{H}, \mathrm{s}), 3.77(6 \mathrm{H}, \mathrm{s}), 3.65$ $(6 \mathrm{H}, \mathrm{s}) ;{ }^{13} \mathrm{C}$ NMR (75 MHz): $\delta 160.3,158.0,131.6,130.2,128.7(\mathrm{t}, \mathrm{J}=5.9 \mathrm{~Hz}), 128.4(\mathrm{t}$, $\mathrm{J}=23.2 \mathrm{~Hz}), 126.4,121.9(\mathrm{t}, \mathrm{J}=282.8 \mathrm{~Hz}), 116.3,103.8,97.6,55.2,54.8,46.6 ;{ }^{19} \mathrm{~F}$ NMR (282 MHz): $\delta$-101.1; LRMS (EI) $m / z$ (relative intensity): $587\left(\mathrm{M}^{+}, 17\right), 585\left(\mathrm{M}^{+}, 16\right)$, 151 (100), 178 (75); HRMS (EI) $m / z$ calculated for $\mathrm{C}_{25} \mathrm{H}_{26} \mathrm{BrF}_{2} \mathrm{NO}_{6} \mathrm{~S}: 585.063227$, found 585.062689 .<smiles>COc1ccc(CN(Cc2ccc(OC)cc2OC)S(=O)(=O)C(F)(F)c2ccc(I)cc2)c(OC)c1</smiles>

$N, N$-Bis(2,4-dimethoxybenzyl)-1,1-difluoro-1-(4-iodophenyl)methanesulfonamide (26): Sulfonamide 16 (5.38 g, 9.00 mmole), NaHMDS (19.8 mL, 19.8 mmole), NSFI (7.09 g, $22.5 \mathrm{mmole})$, THF (360 mL). Flash chromatography (25:75 EtOAc: Hexanes) yields $4.92 \mathrm{~g}(86 \%)$ as a white crystalline solid. M.p. $149-150^{\circ} \mathrm{C} ;{ }^{1} \mathrm{H}$ NMR $(300 \mathrm{MHz}): \delta$ $7.82(2 \mathrm{H}, \mathrm{d}, \mathrm{J}=8.2 \mathrm{~Hz}), 7.37(2 \mathrm{H}, \mathrm{d}, \mathrm{J}=8.3 \mathrm{~Hz}), 7.17(2 \mathrm{H}, \mathrm{d}, \mathrm{J}=8.3 \mathrm{~Hz}), 6.38(2 \mathrm{H}, \mathrm{dd}, \mathrm{J}$ $=8.3,2.0 \mathrm{~Hz}), 6.28(2 \mathrm{H}, \mathrm{d}, \mathrm{J}=2.1 \mathrm{~Hz}), 4.48(4 \mathrm{H}, \mathrm{s}), 3.77(6 \mathrm{H}, \mathrm{s}), 3.65(6 \mathrm{H}, \mathrm{s}),{ }^{13} \mathrm{C} \mathrm{NMR}$ (75 MHz): $\delta$ 160.3, 158.0, 137.6, 130.2, $129.1(\mathrm{t}, \mathrm{J}=23.2 \mathrm{~Hz}), 128.8,128.7,128.6,122.0$ $(\mathrm{t}, \mathrm{J}=282.8 \mathrm{~Hz}), 116.4,103.7,98.8,97.6,55.3,54.8,46.7 ;{ }^{19} \mathrm{~F}$ NMR $(282 \mathrm{MHz}): \delta$ 101.4; LRMS (EI) $m z$ (relative intensity): $656\left(\mathrm{M}^{+}+\mathrm{Na}, 100\right.$ ); HRMS (EI) $m z$ calculated for $\mathrm{C}_{25} \mathrm{H}_{26} \mathrm{~F}_{2} \mathrm{INO}_{6} \mathrm{SNa}$ : 656.0391, found 656.0379 
<smiles>COc1ccc(CN(Cc2ccc(OC)cc2OC)S(=O)(=O)C(F)(F)c2ccc([N+](=O)[O-])cc2)c(OC)c1</smiles>

\section{$N, N$-Bis(2,4-dimethoxybenzyl)-1,1-difluoro-1-(4-nitrophenyl)methanesulfonamide} (27): Same as general procedure except addition of NaHMDS was over 1 hour. Sulfonamide 17 (406 mg, 0.786 mmole), NSFI (620 mg, 1.97 mmole), NaHMDS (1.73 $\mathrm{mL}, 1.73 \mathrm{mmole})$ and THF (31 mL). Flash chromatography (25:75 EtOAc: Hexanes) yielded $282 \mathrm{mg}(60 \%)$ of sulfonamide 27 as a yellow crystalline solid and $86.1 \mathrm{mg}(20 \%)$ of the monofluorinated derivative. M.p. $122-123{ }^{0} \mathrm{C} ;{ }^{1} \mathrm{H}$ NMR $(300 \mathrm{MHz}): \delta 8.32(2 \mathrm{H}, \mathrm{d}, \mathrm{J}$ $=8.7 \mathrm{~Hz}), 7.85(2 \mathrm{H}, \mathrm{d}, \mathrm{J}=8.8 \mathrm{~Hz}), 7.16(2 \mathrm{H}, \mathrm{d}, \mathrm{J}=8.4 \mathrm{~Hz}), 6.39(2 \mathrm{H}, \mathrm{dd}, \mathrm{J}=8.4,2.3$ $\mathrm{Hz}), 6.30(2 \mathrm{H}, \mathrm{d}, \mathrm{J}=2.3 \mathrm{~Hz}), 4.52(4 \mathrm{H}, \mathrm{s}), 3.78(6 \mathrm{H}, \mathrm{s}), 3.67(6 \mathrm{H}, \mathrm{s}) ;{ }^{13} \mathrm{C}$ NMR $(75$ MHz): $\delta 160.5,158.1,149.7,135.7(\mathrm{t}, \mathrm{J}=23.3 \mathrm{~Hz}), 130.3,128.6(\mathrm{t}, \mathrm{J}=5.9 \mathrm{~Hz}), 123.5$, $121.2(\mathrm{t}, \mathrm{J}=283.4 \mathrm{~Hz}), 116.1,103.8,97.7,55.3,54.9,46.8 ;{ }^{19} \mathrm{~F}$ NMR $(282 \mathrm{MHz}): \delta$ 101.6; LRMS (EI) $m z$ (relative intensity): $552\left(\mathrm{M}^{+}, 25\right), 151$ (100), 178 (92); HRMS (EI) $m z$ calculated for $\mathrm{C}_{25} \mathrm{H}_{26} \mathrm{~F}_{2} \mathrm{~N}_{2} \mathrm{O}_{8} \mathrm{~S}: 552.137794$, found 552.137772.<smiles>COc1ccc(CN(Cc2ccc(OC)cc2OC)S(=O)(=O)C(F)(F)c2ccc(C)cc2)c(OC)c1</smiles>

$\mathrm{N}, \mathrm{N}$-Bis(2,4-dimethoxybenzyl)-1,1-difluoro-1-(4-methylphenyl)methanesulfonamide (28): Sulfonamide 18 (135 mg, $0.278 \mathrm{mmole}$ ), NSFI (219 mg, $0.695 \mathrm{mmole})$, [0.9 M] NaHMDS $(680 \mu \mathrm{L}, 0.612 \mathrm{mmole})$ and THF $(11 \mathrm{~mL})$. Flash chromatography (15:85 EtOAc: Hexanes) yielded $110 \mathrm{mg}(76 \%)$ as a clear crystalline solid. Mp $112-113{ }^{\circ} \mathrm{C} ;{ }^{1} \mathrm{H}$ NMR (300 MHz): $\delta 7.55(2 \mathrm{H}, \mathrm{d}, \mathrm{J}=8.1 \mathrm{~Hz}), 7.27(2 \mathrm{H}, \mathrm{d}, \mathrm{J}=8.1 \mathrm{~Hz}), 7.18(2 \mathrm{H}, \mathrm{d}, \mathrm{J}=$ $8.4 \mathrm{~Hz}), 6.37(2 \mathrm{H}, \mathrm{dd}, \mathrm{J}=8.4,2.2 \mathrm{~Hz}), 6.26(2 \mathrm{H}, \mathrm{d}, \mathrm{J}=2.2 \mathrm{~Hz}), 4.47(4 \mathrm{H}, \mathrm{s}), 3.77(6 \mathrm{H}$, s), $3.64(6 \mathrm{H}, \mathrm{s}), 2.41,(3 \mathrm{H}, \mathrm{s}) ;{ }^{13} \mathrm{C} \mathrm{NMR}(75 \mathrm{MHz}): \delta$ 160.2, 158.0, 142.0, 130.3, 129.1, $127.0(\mathrm{t}, \mathrm{J}=6.0 \mathrm{~Hz}), 126.4(\mathrm{t}, \mathrm{J}=22.9 \mathrm{~Hz}), 122.5$ (t, J = 282.3 Hz), 116.6, 103.7, 97.6, 55.2, 54.8, 46.7, 21.4; ${ }^{19} \mathrm{~F}$ NMR (282 MHz): $\delta$-100.3; LRMS (EI) $m / z$ (relative intensity): 
$521\left(\mathrm{M}^{+}, 40\right), 151$ (100), 141 (69), 178 (61); HRMS (EI) $m / z$ calculated for $\mathrm{C}_{26} \mathrm{H}_{29} \mathrm{~F}_{2} \mathrm{NO}_{6} \mathrm{~S}$ : 521.168366 , found 521.167491.<smiles>COc1ccc(CN(Cc2ccc(OC)cc2OC)S(=O)(=O)C(F)(F)c2cccc(Br)c2)c(OC)c1</smiles>

$N, N$-Bis(2,4-dimethoxybenzyl)-1,1-difluoro-1-(3-bromophenyl)methanesulfonamide (29): Sulfonamide 19 (334 mg, 0.607 mmole), [0.9M] NaHMDS (1.5 mL, $1.33 \mathrm{mmole}$ ), NSFI (478 mg, $1.52 \mathrm{mmole})$ and THF (24 mL). Flash chromatography (Benzene) yielded $328 \mathrm{mg}(92 \%)$ as a clear crystalline solid. M.p. $111-113^{\circ} \mathrm{C} ;{ }^{1} \mathrm{H}$ NMR $(300 \mathrm{MHz})$ : $\delta 7.77(1 \mathrm{H}, \mathrm{s}), 7.63(2 \mathrm{H}, \mathrm{dd}, \mathrm{J}=15.7,7.9 \mathrm{~Hz}), 7.34(1 \mathrm{H}, \mathrm{t}, \mathrm{J}=7.9 \mathrm{~Hz}), 7.17(2 \mathrm{H}, \mathrm{d}, \mathrm{J}=$ $8.4 \mathrm{~Hz}), 6.38(2 \mathrm{H}, \mathrm{dd}, \mathrm{J}=8.4,2.2 \mathrm{~Hz}), 6.29(2 \mathrm{H}, \mathrm{d}, \mathrm{J}=2.2 \mathrm{~Hz}), 4.48(4 \mathrm{H}, \mathrm{s}), 3.78(6 \mathrm{H}$, s), $3.67(6 \mathrm{H}, \mathrm{s}) ;{ }^{13} \mathrm{C}$ NMR $(75 \mathrm{MHz}): \delta 160.3,158.0,134.6,131.4(\mathrm{t}, \mathrm{J}=23.2 \mathrm{~Hz}), 130.2$, $130.0(\mathrm{t}, \mathrm{J}=6.3 \mathrm{~Hz}), 129.9,125.8(\mathrm{t}, \mathrm{J}=5.9 \mathrm{~Hz}), 122.3,121.2(\mathrm{t}, \mathrm{J}=283.5 \mathrm{~Hz}), 116.2$, 103.8, 97.6, 55.2, 54.8, 46.7; ${ }^{19} \mathrm{~F}$ NMR (282 MHz): $\delta$-100.9; LRMS (EI) $m z$ (relative intensity): $587\left(\mathrm{M}^{+}, 21\right), 585\left(\mathrm{M}^{+}, 20\right), 151$ (100), 178 (82); HRMS (EI) $m / z$ calculated for $\mathrm{C}_{25} \mathrm{H}_{26} \mathrm{BrF}_{2} \mathrm{NO}_{6} \mathrm{~S}:$ 585.063227, found 585.062784.

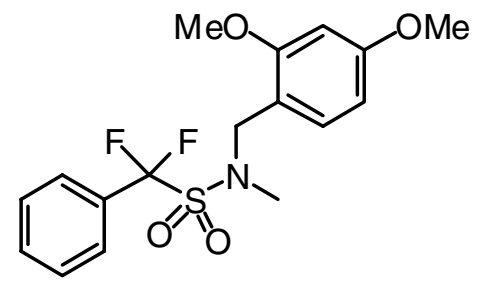

$N$-Methyl- $N$-(2,4-dimethoxybenzyl)-1,1-difluoro-1-phenylmethanesulfonamide (30): Sulfonamide 20 (150.9 mg, 0.448 mmole), [0.9 M] NaHMDS (1.1 mL, 0.985 mmole), NSFI (353 mg, $1.12 \mathrm{mmole})$, THF (18 mL). Flash chromatography (10:90 to 15:85 to 25:75 EtOAc: Hexanes) yielded $105.8 \mathrm{mg}(65 \%)$ as a white solid. M.p. $45-46{ }^{0} \mathrm{C} ;{ }^{1} \mathrm{H}$ NMR (300 MHz): $\delta$ 7.72-7.69 (2H, m), 7.53-7.49 (3H, m), 7.29-7.26 (1H, m), 6.51-6.45 $(2 \mathrm{H}, \mathrm{m}), 4.47(2 \mathrm{H}, \mathrm{bs}), 3.81(6 \mathrm{H}, \mathrm{s}), 2.88(3 \mathrm{H}, \mathrm{s}) ;{ }^{13} \mathrm{C} \mathrm{NMR}(75 \mathrm{MHz}): \delta 160.8,158.6$, 131.7, 130.9, $129.2(\mathrm{t}, \mathrm{J}=24.6 \mathrm{~Hz}), 128.5,127.0(\mathrm{t}, \mathrm{J}=6.1 \mathrm{~Hz}), 122.9$ (t, J = 282.0 Hz), $115.8,104.5,98.3,55.4,55.3,48.3,35.1 ;{ }^{19} \mathrm{~F}$ NMR (282 MHz): $\delta$-100.5; LRMS (EI) $m z$ (relative intensity): $371\left(\mathrm{M}^{+}, 35\right), 151$ (100), $127\left(\mathrm{M}^{+}-\mathrm{SO}_{4} \mathrm{NC}_{10} \mathrm{H}_{14}, 85\right)$; HRMS (EI) $\mathrm{m} z$ calculated for $\mathrm{C}_{17} \mathrm{H}_{19} \mathrm{~F}_{2} \mathrm{NO}_{4} \mathrm{~S}: 371.1003$, found: 371.1015 


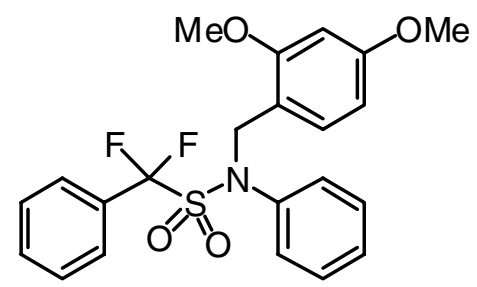

$N$-Phenyl- $N$-(2,4-dimethoxybenzyl)-1,1-difluoro-1-phenylmethanesulfonamide (31): Sulfonamide 21 ( $225 \mathrm{mg}, 0.566 \mathrm{mmole}$ ), NSFI (446 mg, $1.41 \mathrm{mmole}$ ), NaHMDS (1.25 $\mathrm{mL}, 1.25 \mathrm{mmole})$ and THF $(23 \mathrm{~mL})$. Flash chromatography (15:85 EtOAc: Hexanes) yielded $185 \mathrm{mg}(75 \%)$ as a clear crystalline solid. M.p. $138-139^{\circ} \mathrm{C} ;{ }^{1} \mathrm{H}$ NMR $(300 \mathrm{MHz})$ : $\delta 7.69(2 \mathrm{H}, \mathrm{d}, \mathrm{J}=7.2 \mathrm{~Hz}), 7.54-7.46(3 \mathrm{H}, \mathrm{m}), 7.25-7.21(6 \mathrm{H}, \mathrm{m}), 6.38(1 \mathrm{H}, \mathrm{dd}, \mathrm{J}=8.4$, $2.3 \mathrm{~Hz}), 6.25(1 \mathrm{H}, \mathrm{d}, \mathrm{J}=2.3 \mathrm{~Hz}), 4.97(2 \mathrm{H}, \mathrm{s}), 3.75(3 \mathrm{H}, \mathrm{s}), 3.49(3 \mathrm{H}, \mathrm{s}) ;{ }^{13} \mathrm{C}$ NMR $(75$ MHz): $\delta 160.7,158.5,138.2,131.7,131.4,129.7,129.1(\mathrm{t}, \mathrm{J}=22.5 \mathrm{~Hz}), 128.4,128.4$, 128.0, $127.1(\mathrm{t}, \mathrm{J}=6.1 \mathrm{~Hz}), 123.3(\mathrm{t}, \mathrm{J}=283.1 \mathrm{~Hz}), 116.1,104.2,98.0,55.2,55.0,50.9$; ${ }^{19} \mathrm{~F}$ NMR (282 MHz): $\delta$-98.8; LRMS (EI) $m / z$ (relative intensity): 433 (M+, 3), 151 (100), 127 (31); HRMS (EI) $m z$ calculated for $\mathrm{C}_{22} \mathrm{H}_{21} \mathrm{~F}_{2} \mathrm{NO}_{4} \mathrm{~S}: 433.115937$, found 433.117112.<smiles>CN(C)S(=O)(=O)C(F)(F)c1ccccc1</smiles>

$\boldsymbol{N}, \boldsymbol{N}$-Dimethyl-1,1-difluoro-1-phenylmethanesulfonamide (32): Sulfonamide 22 (253 $\mathrm{mg}, 1.27 \mathrm{mmole})$, NSFI (1.00g, $3.18 \mathrm{mmole})$, NaHMDS (2.8 $\mathrm{mL}, 2.8 \mathrm{mmole})$ and THF $(50 \mathrm{~mL})$. Flash chromatography (10:90 EtOAc: Hexanes) yielded $205 \mathrm{mg}(69 \%)$ as a white solid. M.p. $44-45^{0} \mathrm{C} ;{ }^{1} \mathrm{H}$ NMR (300 MHz): $\delta 7.67(2 \mathrm{H}, \mathrm{d}, \mathrm{J}=7.4 \mathrm{~Hz}), 7.55-7.48$ $(3 \mathrm{H}, \mathrm{m}), 3.04(6 \mathrm{H}, \mathrm{s}) ;{ }^{13} \mathrm{C}$ NMR $(75 \mathrm{MHz}): \delta 131.8,129.0(\mathrm{t}, \mathrm{J}=22.4 \mathrm{~Hz}), 128.5,126.9$ $(\mathrm{t}, \mathrm{J}=6.2 \mathrm{~Hz}), 123.3(\mathrm{t}, \mathrm{J}=281.8 \mathrm{~Hz}), 38.3 ;{ }^{19} \mathrm{~F}$ NMR $(282 \mathrm{MHz}): \delta-100.3$; LRMS (CI: $\left.\mathrm{NH}_{3}\right) m z$ (relative intensity): $253\left(\mathrm{M}^{+}+\mathrm{NH}_{4}, 100\right)$; HRMS (EI) $m / z$ calculated for $\mathrm{C}_{7} \mathrm{H}_{5} \mathrm{~F}_{2}$ $\left(\mathrm{M}^{+}-\mathrm{SO}_{2} \mathrm{NC}_{2} \mathrm{H}_{6}\right): 127.0359$, found 127.0363 .

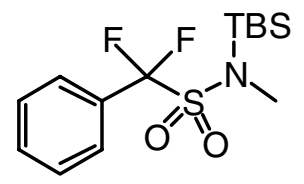

$N$-Methyl- $N$-(dimethyl-tert-butylsilyl)-1,1-difluoro-1-phenylmethanesulfonamide (33): Sulfonamide 23 (228 mg, 0.761 mmole), NaHMDS (1.7 mL, 1.7 mmole), NSFI (600 mg, 1,90 mmole), THF (30 mL). Flash chromatography (15:85 to $25: 75$ to $40: 60$ to 80:20 EtOAc: Hexanes) yielded $170 \mathrm{mg}(67 \%)$ of sulfonamide 33 as a white solid. M.p. $32-34^{\circ} \mathrm{C}$ and $29 \mathrm{mg}(17 \%)$ of sulfonamide $40 ;{ }^{1} \mathrm{H}$ NMR $(300 \mathrm{MHz}): \delta 7.66(2 \mathrm{H}, \mathrm{d}, \mathrm{J}=7.5$ $\mathrm{Hz}), 7.54-7.47(3 \mathrm{H}, \mathrm{m}), 2.99(3 \mathrm{H}, \mathrm{s}), 0.98(9 \mathrm{H}, \mathrm{s}), 0.28(6 \mathrm{H}, \mathrm{s}) ;{ }^{13} \mathrm{C}$ NMR $(75 \mathrm{MHz}): \delta$ 131.6, $129.2(\mathrm{t}, \mathrm{J}=22.7 \mathrm{~Hz}), 128.4,127.1(\mathrm{t}, \mathrm{J}=6.1 \mathrm{~Hz}), 122.1(\mathrm{t}, \mathrm{J}=282.7 \mathrm{~Hz}), 35.3$, 
26.6, 19.7, -3.9; ${ }^{19} \mathrm{~F}$ NMR (282 MHz): $\delta$-100.1; LRMS (EI) $m / z$ (relative intensity): 278 $\left(\mathrm{M}^{+}-\mathrm{C}_{4} \mathrm{H}_{9}, 4\right), 127\left(\mathrm{M}^{+}-\mathrm{SO}_{2} \mathrm{NSiC}_{7} \mathrm{H}_{18}, 100\right), 105$ (49); HRMS (EI) $m / z$ calculated for $\mathrm{C}_{10} \mathrm{H}_{14} \mathrm{NO}_{2} \mathrm{~F}_{2} \mathrm{SSi}\left(\mathrm{M}^{+}-\mathrm{C}_{4} \mathrm{H}_{9}\right): 278.048261$, found: 278.048918 .

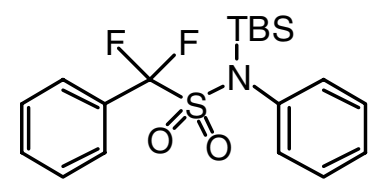

\section{$\boldsymbol{N}$-Phenyl- $\boldsymbol{N}$-(dimethyl-tert-butylsilyl)-1,1-difluoro-1-phenylmethanesulfonamide}

(34): Sulfonamide 24 (231 mg, 0.639 mmole), [0.9M] NaHMDS (3.1 mL, $2.81 \mathrm{mmole}$ ), NSFI (1.00 g, 3.19 mmole), THF ( $25 \mathrm{~mL}$ ). Flash chromatography (5:95 to 25:75 EtOAc: Hexanes) yielded $60.8 \mathrm{mg}$ (24\%) of $\mathbf{3 4}$ as a white solid and $104.5 \mathrm{mg}(58 \%)$ of sulfonamide 41. M.p. $97-99^{\circ} \mathrm{C} ;{ }^{1} \mathrm{H}$ NMR $(300 \mathrm{MHz}): \delta$ 7.62-7.59 $(2 \mathrm{H}, \mathrm{m}), 7.46-7.34(8 \mathrm{H}$, $\mathrm{m}), 0.99(9 \mathrm{H}, \mathrm{s}), 0.26(6 \mathrm{H}, \mathrm{s}) ;{ }^{13} \mathrm{C}$ NMR (300 MHz): $\delta 138.0,131.7,131.6,129.1(\mathrm{t}, \mathrm{J}=$ $22.6 \mathrm{~Hz}), 128.5,128.3,128.1,127.3(\mathrm{t}, \mathrm{J}=6.1 \mathrm{~Hz}), 122.7(\mathrm{t}, \mathrm{J}=285.0 \mathrm{~Hz}), 27.4,20.1$, 2.4; ${ }^{19} \mathrm{~F}$ NMR (282 MHz): $\delta$-96.9; LRMS (EI) $m / z$ (relative intensity): $340\left(\mathrm{M}^{+}-\mathrm{C}_{4} \mathrm{H}_{9}, 10\right.$ ), 127 (100) HRMS (EI) $m / z$ calculated for $\mathrm{C}_{15} \mathrm{H}_{16} \mathrm{~F}_{2} \mathrm{NO}_{2} \mathrm{SSi}\left(\mathrm{M}^{+}-\mathrm{C}_{4} \mathrm{H}_{9}\right): 340.0639$ found: 340.0648 .

\section{General Procedure for the deprotection of Sulfonamides 14 and 35 to 41.}

To a cooled $0^{\circ} \mathrm{C}$ solution of sulfonamide and $\mathrm{CH}_{2} \mathrm{Cl}_{2}$ was added TFA. The reaction mixture immediately turned pink. The reaction was stirred at $0^{\circ} \mathrm{C}$ for $4.5 \mathrm{hrs}$ then the solvent was removed in vacou to yield a pink solid. The solid was suspended in acetone and filtered thru a plug of cotton (acetone rinse). The filtrate was evaporated and the residue purified by flash chromatography (benzene) to yield pure sulfonamides. These could be crystallized further with EtOAc-Hexanes (slow diffusion).<smiles>NS(=O)(=O)C(F)(F)c1ccccc1</smiles>

1,1-Difluoro-1-phenylmethanesulfonamide (13): Sulfonamide 12 (103.8 mg, 0.204 mmole), TFA (1.9 mL), $\mathrm{CH}_{2} \mathrm{Cl}_{2}\left(4.5 \mathrm{~mL}\right.$ ). Flash chromatography (Benzene to 2:98 $\mathrm{Et}_{2} \mathrm{O}$ : Benzene) yielded $38 \mathrm{mg}(90 \%)$ as a clear crystalline solid. M.p. $88-89^{\circ} \mathrm{C} ;{ }^{1} \mathrm{H}$ NMR (acetone- $\left.\mathrm{d}_{6}, 300 \mathrm{MHz}\right): \delta 7.69(2 \mathrm{H}, \mathrm{d}, \mathrm{J}=7.4 \mathrm{~Hz}), 7.63(1 \mathrm{H}, \mathrm{t}, \mathrm{J}=7.2 \mathrm{~Hz}), 7.55(2 \mathrm{H}, \mathrm{t}, \mathrm{J}$ $=7.6 \mathrm{~Hz}), 7.26(2 \mathrm{H}, \mathrm{bs}) ;{ }^{13} \mathrm{C}$ NMR (acetone- $\left.\mathrm{d}_{6}, 75 \mathrm{MHz}\right): \delta 132.6,130.0(\mathrm{t}, \mathrm{J}=22.9 \mathrm{~Hz}$ ), 129.3, $128.0(\mathrm{t}, \mathrm{J}=6.0 \mathrm{~Hz}), 121.4\left(\mathrm{t}, \mathrm{J}=279.4 \mathrm{~Hz}\right.$ ); ${ }^{19} \mathrm{~F}$ NMR (acetone- $\left.{ }_{6}, 282 \mathrm{MHz}\right): \delta$ 103.4; LRMS (EI) $m / z$ (relative intensity): $127\left(\mathrm{M}^{+}-\mathrm{SO}_{2} \mathrm{NH}_{2}, 100\right)$, (CI: $\left.\mathrm{NH}_{3}\right): 225$ 
$\left(\mathrm{M}^{+}+\mathrm{NH}_{4}, 100\right), 207\left(\mathrm{M}^{+}, 1\right)$; HRMS (EI) $m / z$ calculated for $\mathrm{C}_{7} \mathrm{H}_{5} \mathrm{~F}_{2}: 127.0359$ found: 127.0364<smiles>NS(=O)(=O)C(F)(F)c1ccc(Br)cc1</smiles>

1,1-Difluoro-1-(4-bromophenyl)methanesulfonamide (35): Sulfonamide 25 (112.6 mg, $0.192 \mathrm{mmole})$, TFA (1.8 mL), $\mathrm{CH}_{2} \mathrm{Cl}_{2}(4.2 \mathrm{~mL})$. Flash chromatography (Benzene) yielded $50.4 \mathrm{mg}(92) \%$ as a clear crystalline solid. M.p. $138^{\circ} \mathrm{C} ;{ }^{1} \mathrm{H}$ NMR (acetone- $\mathrm{d}_{6}, 300 \mathrm{MHz}$ ): $\delta 7.77(2 \mathrm{H}, \mathrm{d}, \mathrm{J}=8.2 \mathrm{~Hz}), 7.63(2 \mathrm{H}, \mathrm{d}, \mathrm{J}=8.2 \mathrm{~Hz}), 7.32(2 \mathrm{H}, \mathrm{bs}) ;{ }^{13} \mathrm{C}$ NMR (acetone-d ${ }_{6}$, $75 \mathrm{MHz}): \delta 132.6,130.0(\mathrm{t}, \mathrm{J}=6.0 \mathrm{~Hz}), 129.3(\mathrm{t}, \mathrm{J}=23.4 \mathrm{~Hz}), 126.8,121.1(\mathrm{t}, \mathrm{J}=279.8$ $\mathrm{Hz}$ ); ${ }^{19} \mathrm{~F}$ NMR (acetone- $\mathrm{d}_{6}, 282 \mathrm{MHz}$ ): $\delta$-104.0; LRMS (EI) $m / z$ (relative intensity): 285 $\left(\mathrm{M}^{+}, 1\right), 287\left(\mathrm{M}^{+}, 1\right), 205\left(\mathrm{M}^{+}-\mathrm{SO}_{2} \mathrm{NH}_{2}, 100\right), 207\left(\mathrm{M}^{+}-\mathrm{SO}_{2} \mathrm{NH}_{2}, 98\right)$; HRMS (EI) $m / z$ calculated for $\mathrm{C}_{7} \mathrm{H}_{6} \mathrm{BrF}_{2} \mathrm{NO}_{2} \mathrm{~S}: 284.9271$ found 284.9249.

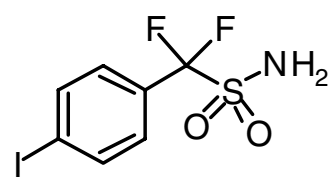

1,1-Difluoro-1-(4-iodophenyl)methanesulfonamide (36): Sulfonamide $26(98.2 \mathrm{mg}$, $0.155 \mathrm{mmole})$, TFA $(1.4 \mathrm{~mL}), \mathrm{CH}_{2} \mathrm{Cl}_{2}(3.4 \mathrm{~mL})$. Flash chromatography (Benzene) yielded $44.5 \mathrm{mg}$ (86\%) as a white crystalline solid. M.p. 152-154 ${ }^{\circ} \mathrm{C}$; ${ }^{1} \mathrm{H}$ NMR (acetone$\left.\mathrm{d}_{6}, 300 \mathrm{MHz}\right): \delta 7.96(2 \mathrm{H}, \mathrm{d}, \mathrm{J}=8.3 \mathrm{~Hz}), 7.47(2 \mathrm{H}, \mathrm{d}, \mathrm{J}=8.3 \mathrm{~Hz}), 7.31(2 \mathrm{H}, \mathrm{bs}) ;{ }^{13} \mathrm{C}$ NMR (acetone- $\left.\mathrm{d}_{6}, 75 \mathrm{MHz}\right): \delta$ 138.6, $129.8(\mathrm{t}, \mathrm{J}=21.5 \mathrm{~Hz}), 129.8(\mathrm{t}, \mathrm{J}=6.0 \mathrm{~Hz}), 121.2$ (t, J $=279.8 \mathrm{~Hz}$ ), 99.2; ${ }^{19} \mathrm{~F}$ NMR (acetone- ${ }_{6}, 282 \mathrm{MHz}$ ): $\delta$-104.2; LRMS (EI) $\mathrm{m} / z$ (relative intensity): $333\left(\mathrm{M}^{+}, 5\right), 253\left(\mathrm{M}^{+}-\mathrm{SO}_{2} \mathrm{NH}_{2}, 100\right), 126$ (50); HRMS (EI) $\mathrm{m} / z$ calculated for $\mathrm{C}_{7} \mathrm{H}_{6} \mathrm{~F}_{2} \mathrm{INO}_{2} \mathrm{~S}: 332.913209$ found 332.913716 .

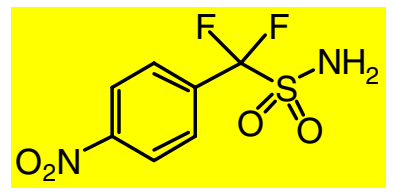

1,1-Difluoro-1-(4-nitrophenyl)methanesulfonamide (37): Sulfonamide 27 (112.8 mg, 0.204 mmole), TFA $(1.9 \mathrm{~mL}), \mathrm{CH}_{2} \mathrm{Cl}_{2}(4.5 \mathrm{~mL})$. Flash chromatography (Benzene) yielded $46.9 \mathrm{mg}(91 \%)$ as a yellow crystalline solid. M.p. $143-144^{\circ} \mathrm{C} ;{ }^{1} \mathrm{H}$ NMR (acetone$\left.\mathrm{d}_{6}, 300 \mathrm{MHz}\right): \delta 8.43(2 \mathrm{H}, \mathrm{d}, \mathrm{J}=8.6 \mathrm{~Hz}), 7.99(2 \mathrm{H}, \mathrm{d}, \mathrm{J}=8.6 \mathrm{~Hz}), 7.48(2 \mathrm{H}, \mathrm{bs}) ;{ }^{13} \mathrm{C}$ NMR (acetone- $\left.\mathrm{d}_{6}, 75 \mathrm{MHz}\right): \delta 151.0,136.1(\mathrm{t}, \mathrm{J}=23.3 \mathrm{~Hz}), 129.8(\mathrm{t}, \mathrm{J}=6.0 \mathrm{~Hz}), 124.5$, 
$120.6\left(\mathrm{t}, \mathrm{J}=280.5 \mathrm{~Hz}\right.$ ); ${ }^{19} \mathrm{~F}$ NMR (acetone-d $\left.\mathrm{d}_{6}, 282 \mathrm{MHz}\right): \delta$-104.3; LRMS (EI) $\mathrm{mz}$ (relative intensity): $172\left(\mathrm{M}^{+}-\mathrm{SO}_{2} \mathrm{NH}_{2}, 100\right)$; HRMS (EI) $\mathrm{m} / z$ calculated for $\mathrm{C}_{7} \mathrm{H}_{4} \mathrm{NO}_{2} \mathrm{~F}_{2}$ $\left(\mathrm{M}^{+}-\mathrm{SO}_{2} \mathrm{NH}_{2}\right): 172.0210$ found: 172.0205<smiles>Cc1ccc(C(F)(F)S(N)(=O)=O)cc1</smiles>

1,1-Difluoro-1-(4-methylphenyl)methanesulfonamide (38): Sulfonamide 28 (86.9 mg, $0.167 \mathrm{mmole})$, TFA $(1.6 \mathrm{~mL}), \mathrm{CH}_{2} \mathrm{Cl}_{2}(3.6 \mathrm{~mL})$. Flash chromatography (Benzene) yielded $20.8 \mathrm{mg}(56 \%)$ as a fine crystalline needles. M.p. $112-113^{\circ} \mathrm{C}$; ${ }^{~} \mathrm{H}$ NMR (acetone$\left.\mathrm{d}_{6}, 300 \mathrm{MHz}\right): \delta 7.56(2 \mathrm{H}, \mathrm{d}, \mathrm{J}=8.0 \mathrm{~Hz}), 7.36(2 \mathrm{H}, \mathrm{d}, \mathrm{J}=8.0 \mathrm{~Hz}), 7.18(2 \mathrm{H}, \mathrm{bs}), 2.41$ $(3 \mathrm{H}, \mathrm{s}) ;{ }^{13} \mathrm{C}$ NMR (acetone- $\left.\mathrm{d}_{6}, 75 \mathrm{MHz}\right): \delta 143.0,129.9,128.0(\mathrm{t}, \mathrm{J}=6.0 \mathrm{~Hz}), 127.1(\mathrm{t}, \mathrm{J}$ $=23.2 \mathrm{~Hz}), 121.6(\mathrm{t}, \mathrm{J}=279.1 \mathrm{~Hz}), 21.3 ;{ }^{19} \mathrm{~F}$ NMR (acetone- $\left.\mathrm{d}_{6}, 282 \mathrm{MHz}\right): \delta-103.1$; LRMS (CI: $\left.\mathrm{NH}_{3}\right) m z$ (relative intensity): $239\left(\mathrm{M}^{+}+\mathrm{NH}_{4}, 100\right)$, (EI): $141\left(\mathrm{M}^{+}-\mathrm{SO}_{2} \mathrm{NH}_{2}\right.$, 100); HRMS (EI) $m / z$ calculated for $\mathrm{C}_{8} \mathrm{H}_{7} \mathrm{~F}_{2}\left(\mathrm{M}^{+}-\mathrm{SO}_{2} \mathrm{NH}_{2}\right)$ : 141.0516 found 141.0514.<smiles>NS(=O)(=O)C(F)(F)c1cccc(Br)c1</smiles>

1,1-Difluoro-1-(3-bromophenyl)methanesulfonamide (39): Sulfonamide 29 (125 mg, $0.213 \mathrm{mmole})$, TFA $(2.0 \mathrm{~mL}), \mathrm{CH}_{2} \mathrm{Cl}_{2}(4.7 \mathrm{~mL})$. Flash chromatography (Benzene) yielded $50 \mathrm{mg}(82 \%)$ as a clear crystalline solid. M.p. $114-115^{\circ} \mathrm{C} ;{ }^{1} \mathrm{H}$ NMR (acetone- $\mathrm{d}_{6}$, $300 \mathrm{MHz}): \delta 7.83-7.82(2 \mathrm{H}, \mathrm{m}), 7.70(1 \mathrm{H}, \mathrm{d}, \mathrm{J}=7.7 \mathrm{~Hz}), 7.54(1 \mathrm{H}, \mathrm{t}, \mathrm{J}=8.0 \mathrm{~Hz}), 7.37$ $(2 \mathrm{H}, \mathrm{bs}) ;{ }^{13} \mathrm{C}$ NMR (acetone- $\left.\mathrm{d}_{6}, 75 \mathrm{MHz}\right): \delta 135.7,132.3(\mathrm{t}, \mathrm{J}=23.4 \mathrm{~Hz}), 131.5,130.7(\mathrm{t}$, $\mathrm{J}=6.2 \mathrm{~Hz}), 127.1(\mathrm{t}, \mathrm{J}=6.0 \mathrm{~Hz}), 122.7,120.4(\mathrm{t}, \mathrm{J}=280.4 \mathrm{~Hz}) ;{ }^{19} \mathrm{~F}$ NMR (acetone- $\mathrm{d}_{6}$, $282 \mathrm{MHz}$ ): $\delta$-103.9; LRMS (EI) $m z$ (relative intensity): $285\left(\mathrm{M}^{+}, 2\right), 287\left(\mathrm{M}^{+}, 2\right), 205$ $\left(\mathrm{M}^{+}-\mathrm{SO}_{2} \mathrm{NH}_{2}, 100\right), 207\left(\mathrm{M}^{+}-\mathrm{SO}_{2} \mathrm{NH}_{2}, 98\right)$; HRMS (EI) $m / z$ calculated for $\mathrm{C}_{7} \mathrm{H}_{6} \mathrm{O}_{2} \mathrm{~N}^{79} \mathrm{BrF}_{2} \mathrm{~S}$ : 284.9271, found: 284.9259<smiles>CNS(=O)(=O)C(F)(F)c1ccccc1</smiles>

$\mathrm{N}$-Methyl-1,1-difluoro-1-phenylmethanesulfonamide (40): Route A same as general procedure: Sulfonamide 33 (43.5 $\mathrm{mg}, 0.117 \mathrm{mmole})$, TFA (1.1 mL), $\mathrm{CH}_{2} \mathrm{Cl}_{2}(2.6 \mathrm{~mL})$. Flash chromatography (15:85 EtOAc:Hexanes) yielded $23.4 \mathrm{mg}$ (90\%) as a clear colorless oil. Route B A solution of sulfonamide 33 (96.2 mg, $0.286 \mathrm{mmole}), 1 \mathrm{~N} \mathrm{HCl}$ $(3.8 \mathrm{~mL})$ and THF $(3.8 \mathrm{~mL})$ was stirred at $\mathrm{rt}$ overnight. The mixture was diluted with $\mathrm{Et}_{2} \mathrm{O}$, washed with aq. $\mathrm{NaHCO}_{3}$, brine, dried over $\mathrm{MgSO}_{4}$, filtered and concentrated. 
Flash chromatography (15:85 EtOAc:Hexanes) yielded $55.2 \mathrm{mg}$ (87\%); ${ }^{1} \mathrm{H}$ NMR (300 MHz): $\delta 7.69(2 \mathrm{H}, \mathrm{d}, \mathrm{J}=7.5 \mathrm{~Hz}), 7.57-7.47(3 \mathrm{H}, \mathrm{m}), 4.58(1 \mathrm{H}, \mathrm{bs}), 2.97(3 \mathrm{H}, \mathrm{d}, \mathrm{J}=4.9$ $\mathrm{Hz}) ;{ }^{13} \mathrm{C}$ NMR $(75 \mathrm{MHz}): \delta 131.9,128.5,128.5(\mathrm{t}, \mathrm{J}=16.9 \mathrm{~Hz}), 127.1(\mathrm{t}, \mathrm{J}=6.0 \mathrm{~Hz})$, $121.7\left(\mathrm{t}, \mathrm{J}=281.6 \mathrm{~Hz}\right.$ ), 30.4; ${ }^{19} \mathrm{~F}$ NMR (282 MHz): $\delta-101.2$; LRMS (EI) $m z$ (relative intensity): $127\left(\mathrm{M}^{+}-\mathrm{SO}_{2} \mathrm{NH}_{2}, 100\right)$; $\left(\mathrm{CI}: \mathrm{NH}_{3}\right): 239\left(\mathrm{M}^{+}+\mathrm{NH}_{4}, 100\right)$; HRMS (EI) $m / z$ calculated for $\mathrm{C}_{7} \mathrm{H}_{5} \mathrm{~F}_{2}: 127.0359$, found: 127.0361

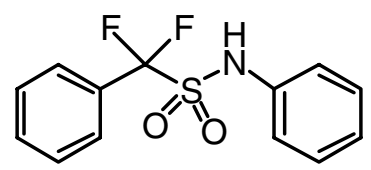

1,1-Difluoro-N,1-diphenylmethanesulfonamide (41): Route A same as general procedure, reaction time 1.75 hours: Sulfonamide 31 (73.3 mg, 0.169 mmole), TFA $(530 \mu \mathrm{L}), \mathrm{CH}_{2} \mathrm{Cl}_{2}(4.77 \mathrm{~mL})$. Flash chromatography (25:75 EtOAc:Hexanes) yielded $45.9 \mathrm{mg}(96 \%)$ as a white crystalline solid. Route B A solution of sulfonamide 34 (60.0 $\mathrm{mg}, 0.15 \mathrm{mmole}), 0.12 \mathrm{~N} \mathrm{HCl}(2 \mathrm{~mL})$ and THF $(2 \mathrm{~mL})$ was stirred at room temperature for 2.5 hours. The mixture was diluted with $\mathrm{Et}_{2} \mathrm{O}$, washed with aq. $\mathrm{NaHCO}_{3}$, brine, dried over $\mathrm{MgSO}_{4}$ and concentrated. Flash chromatography (25:75 EtOAc:Hexanes) yielded $33.2 \mathrm{mg}(78 \%)$. M.p. $70-71^{\circ} \mathrm{C}$; ${ }^{1} \mathrm{H}$ NMR $(300 \mathrm{MHz}): \delta 7.65(2 \mathrm{H}, \mathrm{d}, \mathrm{J}=7.7 \mathrm{~Hz}), 7.57-$ 7.52 (1H, m), 7.48-7.43 (2H, m), 7.37-7.32 (2H, m), 7.28-7.20 (3H, m), $6.85(1 \mathrm{H}, \mathrm{bs})$; ${ }^{13} \mathrm{C}$ NMR (75 MHz): $\delta$ 135.5, 132.2, 129.3, 128.6, $127.9(\mathrm{t}, \mathrm{J}=22.4 \mathrm{~Hz}), 127.2(\mathrm{t}, \mathrm{J}=6.1$ $\mathrm{Hz}), 126.3,122.4,122.0$ (t, J = 283.9 Hz); ${ }^{19} \mathrm{~F}$ NMR (282 MHz): $\delta$-99.8; LRMS (EI) $m / z$ (relative intensity): $283\left(\mathrm{M}^{+}, 2\right), 127$ (100), 219 (32); HRMS (EI) $m / z$ calculated for $\mathrm{C}_{13} \mathrm{H}_{11} \mathrm{~F}_{2} \mathrm{NO}_{2} \mathrm{~S}: 283.047857$ found 283.048080.

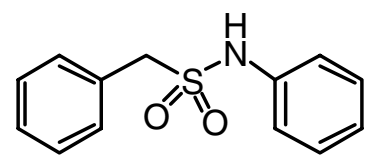

N,1-Diphenylmethanesulfonamide (42): Aniline (2.76 g, 14.1 mmole) was added to a $0^{\circ} \mathrm{C}$ solution of benzylsulfonyl chloride $(2.69 \mathrm{~g}, 14.1 \mathrm{mmole})$ and THF $(30 \mathrm{~mL})$. The reaction was gradually warmed to room temperature and stirred overnight. The reaction was filtered and washed with EtOAc. The filtrate was washed with aq. $\mathrm{NH}_{4} \mathrm{Cl}$, brine, dried over $\mathrm{MgSO}_{4}$, and concentrated to dryness. Flash chromatography (25:75 EtOAc:Hexanes) yielded 2.33g (87\%) as a off-white crystalline solid. Mp $99^{\circ} \mathrm{C}$ (lit 98$\left.99^{\circ} \mathrm{C}\right)^{4}{ }^{1} \mathrm{H}$ NMR: $\delta$ 7.37-7.32 $(5 \mathrm{H}, \mathrm{m}), 7.26-7.24(2 \mathrm{H}, \mathrm{m}), 7.19-7.13(3 \mathrm{H}, \mathrm{m}), 6.47(1 \mathrm{H}$, bs), $4.32(2 \mathrm{H}, \mathrm{s}) ;{ }^{13} \mathrm{C}$ NMR: $\delta 136.9,130.7,129.5,128.8,128.7,128.3,124.6,119.7,57.2$.<smiles>CNS(=O)(=O)Cc1ccccc1</smiles> 
$N$-Methyl-1-phenylmethanesulfonamide (43): Prepared with slight variation of known literature procedures. ${ }^{5}$ Benzylsulfonyl chloride (2.75 g, $\left.14.4 \mathrm{mmole}\right)$, [2.0M] methylamine $(15.1 \mathrm{~mL}, 30.3 \mathrm{mmole})$ in THF $(15 \mathrm{~mL})$. Recrystallization from hot ethanol yielded $1.98 \mathrm{~g}(74 \%)$ as a white crystalline solid. Mp $107-108^{0} \mathrm{C}$ (lit $108-112^{\circ} \mathrm{C}$ ) ${ }^{1} \mathrm{H}$ NMR: $\delta 7.35(5 \mathrm{H}, \mathrm{bs}), 4.57(1 \mathrm{H}, \mathrm{bd}, \mathrm{J}=4.1 \mathrm{~Hz}), 4.20(2 \mathrm{H}, \mathrm{s}), 2.62(3 \mathrm{H}, \mathrm{d}, \mathrm{J}=5.0 \mathrm{~Hz})$; ${ }^{13} \mathrm{C}$ NMR: $\delta 130.5,129.2,128.7,128.5,57.4,29.6$.

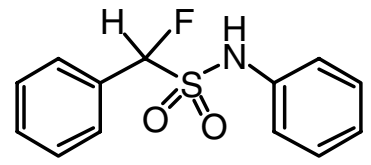

1-Fluoro- $N, 1$-diphenylmethanesulfonamide (44): To a solution of sulfonamide 42 (155 $\mathrm{mg}, 0.627 \mathrm{mmole})$ and THF $(18 \mathrm{~mL})$ at $-78^{\circ} \mathrm{C}$ was added $[1.15 \mathrm{M}] n$-BuLi $(1.2 \mathrm{~mL}, 1.38$ mmole). The reaction was warmed to $0^{\circ} \mathrm{C}$ for 1 hour before being cooled to $-78^{\circ} \mathrm{C}$. A solution of NSFI (494 mg, 1.57 mmole) in THF (5 mL $+2 \mathrm{~mL}$ rinse) was added via cannula. The reaction was stirred at $-78^{\circ} \mathrm{C}$ for 0.5 hours, then warmed to room temperature and stirred for 2 hours further. The reaction was quenched with aq. $\mathrm{NH}_{4} \mathrm{Cl}$ and extracted with EtOAc (x3), washed with $\mathrm{NaHCO}_{3}$, brine, dried over $\mathrm{MgSO}_{4}$ and concentrated. Flash chromatography (15:85 EtOAc:Hexanes) yielded $52.5 \mathrm{mg}(32 \%)$ as a white crystalline solid. M.p. $93-94{ }^{0} \mathrm{C} ;{ }^{1} \mathrm{H}$ NMR $(300 \mathrm{MHz}): \delta$ 7.52-7.50 $(2 \mathrm{H}, \mathrm{m}), 7.46-$ $7.34(5 \mathrm{H}, \mathrm{m}), 7.27-7.23(3 \mathrm{H}, \mathrm{m}), 6.96(1 \mathrm{H}, \mathrm{bs}), 6.05(1 \mathrm{H}, \mathrm{d}, \mathrm{J}=45.7 \mathrm{~Hz}) ;{ }^{13} \mathrm{C} \mathrm{NMR}(75$ MHz): $\delta 135.7,130.8,129.6,128.7,128.7(\mathrm{~d}, \mathrm{~J}=19.6 \mathrm{~Hz}), 127.7(\mathrm{~d}, \mathrm{~J}=6.5 \mathrm{~Hz}), 126.1$, 122.1, 99.1 (d, J = 216.4 Hz); ${ }^{19} \mathrm{~F}$ NMR (282 MHz): $\delta-172.7(\mathrm{~d}, \mathrm{~J}=45.7 \mathrm{~Hz})$; LRMS (EI) $m / z$ (relative intensity): $265\left(\mathrm{M}^{+}, 4\right), 109\left(\mathrm{M}^{+}-\mathrm{SO}_{2} \mathrm{NC}_{6} \mathrm{H}_{6}, 100\right)$; HRMS (EI) $m z$ calculated for $\mathrm{C}_{13} \mathrm{H}_{12} \mathrm{FNO}_{2} \mathrm{~S}$ : 265.0573, found: 265.0572

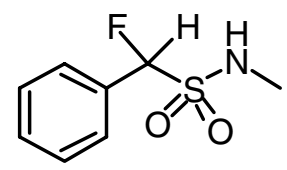

$\mathrm{N}$-Methyl-1-fluoro-1-phenylmethanesulfonamide (45): To a solution of sulfonamide 43 (99.0 $\mathrm{mg}, 0.534 \mathrm{mmole})$ and THF $(15 \mathrm{~mL})$ at $-78^{\circ} \mathrm{C}$ was added [1.36M] $n$-BuLi (865 $\mu \mathrm{L}, 1.17$ mmole). The reaction was warmed to $0^{\circ} \mathrm{C}$ for 1 hour before being cooled to $78^{\circ} \mathrm{C}$. A solution of NSFI (421 mg, $\left.1.34 \mathrm{mmole}\right)$ in THF (5 mL $+1 \mathrm{~mL}$ rinse) was added via cannula. The reaction was stirred at $-78^{\circ} \mathrm{C}$ for 1.5 hours, then warmed to room temperature and stirred for 2 hours further. The reaction was quenched with aq. $\mathrm{NH}_{4} \mathrm{Cl}$ and extracted with EtOAc (x3), washed with $\mathrm{NaHCO}_{3}$, brine, dried over $\mathrm{MgSO}_{4}$ and concentrated. Flash chromatography (5:95 to 10:90 to 15:85 EtOAc:Hexanes) yielded $11.7 \mathrm{mg}(11 \%)$ of sulfonamide 45 as a white solid, and $19.1 \mathrm{mg}(10 \%)$ of sulfonamide 46; Mp 59-60 ${ }^{0}$; ${ }^{1} \mathrm{H}$ NMR (300 MHz): $\delta$ 7.57-7.54 (2H, m), 7.48-7.43 (3H, m), $6.10(1 \mathrm{H}$, $\mathrm{d}, \mathrm{J}=45.7 \mathrm{~Hz}), 4.51(1 \mathrm{H}, \mathrm{bs}), 2.79(3 \mathrm{H}, \mathrm{d}, \mathrm{J}=4.9 \mathrm{~Hz}) ;{ }^{13} \mathrm{C}$ NMR $(75 \mathrm{MHz}): \delta 130.5(\mathrm{~d}, \mathrm{~J}$ $=1.3 \mathrm{~Hz}), 129.8,(\mathrm{~d}, \mathrm{~J}=19.6 \mathrm{~Hz}), 128.6,127.3(\mathrm{~d}, \mathrm{~J}=6.7 \mathrm{~Hz}), 100.8(\mathrm{~d}, \mathrm{~J}=214.9 \mathrm{~Hz})$, 
30.1; ${ }^{19} \mathrm{~F}$ NMR (282 MHz): $\delta$-175.5; LRMS (CI: $\mathrm{NH}_{3}$ ) $m / z$ (relative intensity): 221 $\left(\mathrm{M}^{+}+\mathrm{NH}_{4}, 100\right)$; HRMS (EI) $m / z$ calculated for $\mathrm{C}_{7} \mathrm{H}_{6} \mathrm{~F}\left(\mathrm{M}^{+}-\mathrm{SO}_{2} \mathrm{NCH}_{4}\right): 109.0454$, found: 109.0456 .<smiles>CN(S(=O)(=O)c1ccccc1)S(=O)(=O)c1ccccc1</smiles>

$N$-\{[fluoro(phenyl)methyl]sulfonyl $\}-N$-methylbenzenesulfonamide $\quad$ (46): $\quad$ See procedure for sulfonamide 45; white crystalline solid. Mp $112-113{ }^{0} \mathrm{C}$; ${ }^{1} \mathrm{H}$ NMR (300 $\mathrm{MHz}): \delta$ 8.05-8.02 $(2 \mathrm{H}, \mathrm{m}), 7.68-7.63(3 \mathrm{H}, \mathrm{m}), 7.59-7.49(5 \mathrm{H}, \mathrm{m}), 6.75(1 \mathrm{H}, \mathrm{d}, \mathrm{J}=45.4$ $\mathrm{Hz}), 3.23$ (3H, s); ${ }^{13} \mathrm{C}$ NMR (75 MHz): $\delta$ 138.3, 134.3, 131.3, 129.3, 128.8, 128.2, 128.1, 127.9, 103.7 (d, J = 219.8 Hz), 35.9; ${ }^{19} \mathrm{~F}$ NMR (282 MHz): $\delta$-173.0; LRMS (CI: $\left.\mathrm{NH}_{3}\right) \mathrm{mz}$ (relative intensity): $361\left(\mathrm{M}^{+}+\mathrm{NH}_{4}, 100\right)$; HRMS (EI) $m / z$ calculated for $\mathrm{C}_{7} \mathrm{H}_{6} \mathrm{~F}\left(\mathrm{M}^{+}-\right.$ $\left.\mathrm{S}_{2} \mathrm{O}_{4} \mathrm{NC}_{7} \mathrm{H}_{8}\right): 109.0454$, found: 109.0452<smiles>CCS(=O)(=O)N(Cc1ccc(OC)cc1OC)Cc1ccc(OC)cc1OC</smiles>

$N, N$-Bis(2,4-dimethoxybenzyl)-ethanesulfonamide (47): To a cooled $0^{\circ} \mathrm{C}$ solution of ethanesulfonyl chloride (780 mg, $6.07 \mathrm{mmole})$ and THF $(6 \mathrm{~mL})$ was added a solution of amine 6 (1.75 g, $5.52 \mathrm{mmole})$, triethylamine (670 mg, $6.62 \mathrm{mmole})$ and THF (10 mL) over 1 hour. The reaction was removed from the ice bath and stirred overnight. The reaction mixture was filtered, washed with EtOAc, and concentrated to dryness. Flash chromatography (25:75 EtOAc:Hexanes) yielded $1.73 \mathrm{~g}(77 \%)$ as a white solid. Mp 84$85^{0} \mathrm{C}{ }^{1} \mathrm{H}$ NMR: $\delta 7.21(2 \mathrm{H}, \mathrm{d}, \mathrm{J}=8.1 \mathrm{~Hz}), 6.46-6.42(4 \mathrm{H}, \mathrm{m}), 4.38(4 \mathrm{H}, \mathrm{s}), 3.79(6 \mathrm{H}, \mathrm{s})$, $3.75(6 \mathrm{H}, \mathrm{s}), 2.86(2 \mathrm{H}, \mathrm{q}, \mathrm{J}=7.4 \mathrm{~Hz}), 1.23(3 \mathrm{H}, \mathrm{t}, \mathrm{J}=7.4 \mathrm{~Hz}) ;{ }^{13} \mathrm{C} \mathrm{NMR}: \delta 160.3,158.2$, 130.7, 117.0, 103.8, 98.0, 55.2, 54.9, 47.3, 45.2, 7.9; LRMS (EI) $m z$ (relative intensity): $409\left(\mathrm{M}^{+}, 9\right), 151$ (100); HRMS (EI) $m / z$ calculated for $\mathrm{C}_{20} \mathrm{H}_{27} \mathrm{NO}_{6} \mathrm{~S}: 409.155910$, found 409.155880 . 


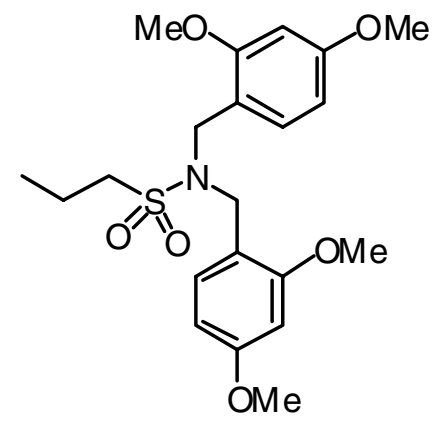

$N, N$-Bis(2,4-dimethoxybenzyl)-propane-1-sulfonamide (48): To a cooled $0^{\circ} \mathrm{C}$ solution of 1-propanesulfonyl chloride $(588 \mathrm{mg}, 4.12 \mathrm{mmole})$ and THF $(4 \mathrm{~mL})$ was added a solution of amine 6 ( $1.19 \mathrm{~g}, 3.75 \mathrm{mmole})$, triethylamine ( $455 \mathrm{mg}, 4.50 \mathrm{mmole})$ and THF $(7 \mathrm{~mL})$ over 1 hour. The reaction was removed from the ice bath and stirred overnight. The reaction mixture was filtered, washed with EtOAc, and concentrated to dryness. Flash chromatography (25:75 EtOAc:Hexanes) yielded $1.25 \mathrm{~g}$ (79\%) as a white solid. Mp 88-90 $0^{\circ} \mathrm{C} ;{ }^{1} \mathrm{H}$ NMR: $\delta 7.22(2 \mathrm{H}, \mathrm{d}, \mathrm{J}=8.3 \mathrm{~Hz}), 6.47-6.42(4 \mathrm{H}, \mathrm{m}), 4.37(4 \mathrm{H}, \mathrm{s}), 3.80$ $(6 \mathrm{H}, \mathrm{s}), 3.76(6 \mathrm{H}, \mathrm{s}), 2.83-2.78(2 \mathrm{H}, \mathrm{m}), 1.74(2 \mathrm{H}$, sextet, J = $7.7 \mathrm{~Hz}), 0.93(3 \mathrm{H}, \mathrm{t}, \mathrm{J}=7.4$ $\mathrm{Hz}){ }^{13} \mathrm{C}$ NMR: $\delta 160.4,158.3,130.8,117.1,103.9,98.2,55.3,55.0,54.9,45.2,17.0$, 13.0; LRMS (EI) $m z$ (relative intensity): 423 (M+11), 151 (100), 178 (45); HRMS (EI) $m z$ calculated for $\mathrm{C}_{21} \mathrm{H}_{29} \mathrm{NO}_{6} \mathrm{~S}: 423.171560$, found 423.171726 .<smiles>COc1ccc(CN(Cc2ccc(OC)cc2OC)S(=O)(=O)C(C)C)c(OC)c1</smiles>

$\boldsymbol{N}, \boldsymbol{N}$-Bis(2,4-dimethoxybenzyl)-propane-2-sulfonamide (49): To a cooled $-78^{\circ} \mathrm{C}$ solution of sulfonamide 47 (178.3 mg, $0.435 \mathrm{mmole})$ and THF $(5 \mathrm{~mL})$ was added [1.15 M] $n$-BuLi $\left(450 \mu \mathrm{L}, 0.522\right.$ mmole). After $1 \mathrm{hr}$ at $-78^{\circ} \mathrm{C}$, iodomethane $(38 \mu \mathrm{L}, 0.609$ mmole) was added. The reaction was warmed to room temperature and stirred overnight. The reaction was quenched with aq. $\mathrm{NH}_{4} \mathrm{Cl}$, the product extracted with EtOAc (x3), washed with aq. $\mathrm{NaHCO}_{3}$, brine, dried over $\mathrm{MgSO}_{4}$, filtered and concentrated to dryness. Flash chromatography (15:85 to 25:75 EtOAc: Hexanes) yielded $139 \mathrm{mg}(76 \%)$ as a colorless oil; ' ${ }^{1} \mathrm{H}$ NMR: $\delta 7.21(2 \mathrm{H}, \mathrm{d}, \mathrm{J}=8.2 \mathrm{~Hz}), 6.46-6.41(4 \mathrm{H}, \mathrm{m}), 4.37(4 \mathrm{H}, \mathrm{s}), 3.80$ $(6 \mathrm{H}, \mathrm{s}), 3.75(6 \mathrm{H}, \mathrm{s}), 2.95(1 \mathrm{H}$, quintet, $\mathrm{J}=6.8 \mathrm{~Hz}), 1.26(6 \mathrm{H}, \mathrm{d}, \mathrm{J}=6.8 \mathrm{~Hz}) ;{ }^{13} \mathrm{C}$ NMR: $\delta$ 160.3, 158.3, 130.9, 117.4, 103.9, 98.1, 55.3, 55.0, 54.1, 45.8, 16.5; LRMS (EI) $\mathrm{m} / z$ (relative intensity): $423\left(\mathrm{M}^{+}, 17\right), 151$ (100); HRMS (EI) $\mathrm{w} / z$ calculated for $\mathrm{C}_{21} \mathrm{H}_{29} \mathrm{NO}_{6} \mathrm{~S}$ : 423.1716 found: 423.1718 . 


\section{General Procedure for the fluorination of alkyl sulfonamides 50 to 55}

To a $-78^{\circ} \mathrm{C}$ solution of sulfonamide (or mono-fluorosulfonamide) (1.0 eq), freshly distilled HMPA (5.0 eq) and THF was added $n$-BuLi (1.3 eq.) dropwise. The resulting darken solution was stirred at $-78^{\circ} \mathrm{C}$ for hr, before a solution of NSFI (1.5 eq.) in THF was added via cannula. The reaction was stirred at $-78^{\circ} \mathrm{C}$ for $3 \mathrm{hrs}$ before being warmed to rt and stirred overnight. The mixture was quenched with aq $\mathrm{NH}_{4} \mathrm{Cl}$, extracted with EtOAc (x3), washed with aq $\mathrm{NaHCO}_{3}$, brine, dried, and concentrated. Purification via flash chromatography (100\% benzene or 10:90 to 15:85 EtOAc: Hexanes) yielded pure sulfonamides.

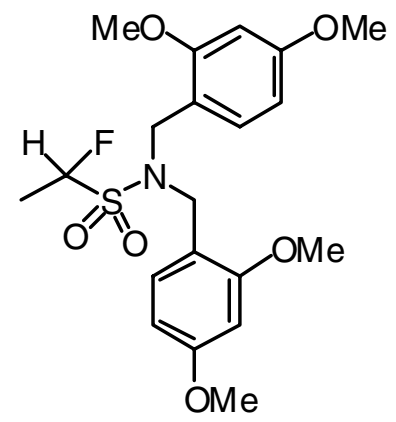

$\mathbf{N}, \boldsymbol{N}$-Bis(2,4-dimethoxybenzyl)-1-fluoro-ethanesulfonamide (50): Sulfonamide 47 (308 $\mathrm{mg}, 0.752 \mathrm{mmole}),[1.43 \mathrm{M}] n$-BuLi $(630 \mu \mathrm{L}, 0.903 \mathrm{mmole}), \mathrm{HMPA}(655 \mu \mathrm{L}, 3.76$ mmole), THF (21 mL) and NSFI (356 $\mathrm{mg}, 1.13 \mathrm{mmole})$ in THF $(6 \mathrm{~mL}+3 \mathrm{~mL}$ rinse). Flash chromatography (15:85 to 25:75 EtOAc:Hexanes) yielded $192.4 \mathrm{mg}(60 \%)$ as a pale yellow oil; ${ }^{1} \mathrm{H}$ NMR $(300 \mathrm{MHz}): \delta 7.20(2 \mathrm{H}, \mathrm{d}, \mathrm{J}=8.2 \mathrm{~Hz}), 6.46-6.41(4 \mathrm{H}, \mathrm{m}), 4.96$ $(1 \mathrm{H}, \mathrm{dq}, \mathrm{J}=47.9,6.3 \mathrm{~Hz}), 4.51(2 \mathrm{H}, \mathrm{d}, \mathrm{J}=15.6 \mathrm{~Hz}), 4.34(2 \mathrm{H}, \mathrm{d}, \mathrm{J}=15.6 \mathrm{~Hz}), 3.79(6 \mathrm{H}$, s), $3.74(6 \mathrm{H}, \mathrm{s}), 1.65(3 \mathrm{H}, \mathrm{dd}, \mathrm{J}=23.5,6.3 \mathrm{~Hz}) ;{ }^{13} \mathrm{C} \mathrm{NMR}(75 \mathrm{MHz}): \delta 160.4,158.3$, 130.6, 116.8, 103.9, $98.4(\mathrm{~d}, \mathrm{~J}=210.5 \mathrm{~Hz}), 98.1,55.3,55.0,45.8,15.3(\mathrm{~d}, \mathrm{~J}=21.0 \mathrm{~Hz}$ ); ${ }^{19}$ F NMR $(282 \mathrm{MHz}): \delta-172.1(\mathrm{dq}, \mathrm{J}=47.9,23.5 \mathrm{~Hz}$ ); LRMS (EI) $m z$ (relative intensity): 427 ( $\left.\mathrm{M}^{+}, 40\right), 151$ (100), 178 (77); HRMS (EI) $m z$ calculated for $\mathrm{C}_{20} \mathrm{H}_{26} \mathrm{FNO}_{6} \mathrm{~S}$ : 427.146488, found 427.146297. 


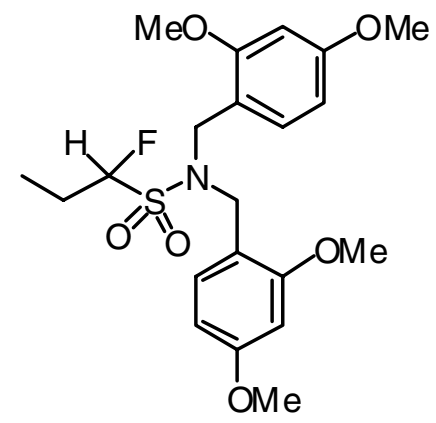

$\mathrm{N}, \mathrm{N}$-Bis(2,4-dimethoxybenzyl)-1-fluoro-propane-1-sulfonamide (51): Sulfonamide 48 (107.8 mg, 0.254 mmole), [1.15 M] $n$-BuLi $(265 \mu \mathrm{L}, 0.305$ mmole), HMPA $(220 \mu \mathrm{L}$, $1.27 \mathrm{mmole})$, in THF (7 mL) and NSFI (120 mg, $0.381 \mathrm{mmole})$ in THF $(2 \mathrm{~mL}+1 \mathrm{~mL})$. Flash chromatography (15:85 EtOAc:Hexanes) yielded $77.3 \mathrm{mg}(69 \%)$ as a pale yellow oil; ${ }^{1} \mathrm{H}$ NMR (300 MHz): $\delta 7.20(2 \mathrm{H}, \mathrm{d}, \mathrm{J}=8.2 \mathrm{~Hz}), 6.46-6.41(4 \mathrm{H}, \mathrm{m}), 4.78(1 \mathrm{H}, \mathrm{ddd}, \mathrm{J}$ $=48.3,8.7,3.8 \mathrm{~Hz}), 4.53(2 \mathrm{H}, \mathrm{d}, \mathrm{J}=15.7 \mathrm{~Hz}), 4.32(2 \mathrm{H}, \mathrm{d}, \mathrm{J}=15.7 \mathrm{~Hz}), 3.79(6 \mathrm{H}, \mathrm{s})$, $3.74(6 \mathrm{H}, \mathrm{s}), 2.11-1.96(2 \mathrm{H}, \mathrm{m}), 1.06(3 \mathrm{H}, \mathrm{t}, \mathrm{J}=7.4 \mathrm{~Hz}) ;{ }^{13} \mathrm{C}$ NMR $(75 \mathrm{MHz}): \delta 160.4$, 158.3, 130.6, 116.8, 103.9, $102.5(\mathrm{~d}, \mathrm{~J}=211.1 \mathrm{~Hz}), 98.1,55.3,55.0,45.7,22.9(\mathrm{~d}, \mathrm{~J}=$ $20.1 \mathrm{~Hz}), 9.1(\mathrm{~d}, \mathrm{~J}=3.9 \mathrm{~Hz}) ;{ }^{19} \mathrm{~F}$ NMR $(282 \mathrm{MHz}): \delta-180.0(\mathrm{ddd}, \mathrm{J}=49.3,30.2,20.0$ Hz); LRMS (EI) $m z$ (relative intensity): 441 (M+2 28), 151 (100), 178 (74); HRMS (EI) $m / z$ calculated for $\mathrm{C}_{21} \mathrm{H}_{28} \mathrm{FNO}_{6} \mathrm{~S}: 441.162138$ found 441.161716.<smiles>COc1ccc(CN(Cc2ccc(OC)cc2OC)S(=O)(=O)C(C)(C)F)c(OC)c1</smiles>

$\mathrm{N}, \mathrm{N}$-Bis(2,4-dimethoxybenzyl)-2-fluoro-propane-2-sulfonamide (52): To a $-78^{\circ} \mathrm{C}$ solution of $t$-BuOK (56 mg, 0.497 mmole), HMPA (200 $\mu \mathrm{L}, 1.13 \mathrm{mmole})$ and THF (3 $\mathrm{mL})$ was added $[1.15 \mathrm{M}] n$-BuLi $(432 \mu \mathrm{L}, 0.497 \mathrm{mmole})$. After $1 \mathrm{hr}$ at $-78^{\circ} \mathrm{C}$ a solution of sulfonamide 49 ( $95.7 \mathrm{mg}, 0.226 \mathrm{mmole})$ and THF ( $2 \mathrm{~mL}+1 \mathrm{~mL}$ rinse) was added via cannula. After $1 \mathrm{hr}$ further at $-78^{\circ} \mathrm{C}$, a solution of NSFI ( $\left.214 \mathrm{mg}, 0.678 \mathrm{mmole}\right)$ and THF $\left(2 \mathrm{~mL}+1 \mathrm{~mL}\right.$ rinse) was added via cannula. The reaction was stirred for $2 \mathrm{hr}$ at $-78^{\circ} \mathrm{C}$ and warmed to room temperature and stirred overnight. The reaction was quenched with aq. $\mathrm{NH}_{4} \mathrm{Cl}$, extracted with EtOAc (x3), washed with aq. $\mathrm{NaHCO}_{3}$, brine, dried over $\mathrm{MgSO}_{4}$, filtered and concentrated to dryness. Flash chromatography (15:85 EtOAc:Hexanes) yielded $41.2 \mathrm{mg}(41 \%)$ as a pale yellow oil; ${ }^{1} \mathrm{H}$ NMR $(300 \mathrm{MHz}): \delta$ $7.21(2 \mathrm{H}, \mathrm{d}, \mathrm{J}=8.4 \mathrm{~Hz}), 6.39(2 \mathrm{H}, \mathrm{dd}, \mathrm{J}=8.4,2.3 \mathrm{~Hz}), 6.29(2 \mathrm{H}, \mathrm{d}, \mathrm{J}=2.3 \mathrm{~Hz}), 4.45$ $(4 \mathrm{H}, \mathrm{s}), 3.77(6 \mathrm{H}, \mathrm{s}), 3.63(6 \mathrm{H}, \mathrm{s}), 1.74(6 \mathrm{H}, \mathrm{d}, \mathrm{J}=21.2 \mathrm{~Hz}) ;{ }^{13} \mathrm{C} \mathrm{NMR}(75 \mathrm{MHz}): \delta$ 
160.2, 158.2, 130.4, 117.2, $108.1(\mathrm{~d}, \mathrm{~J}=210.4 \mathrm{~Hz}), 103.9,97.8,55.3,54.9,46.3,22.5$ (d, $\mathrm{J}=22.5 \mathrm{~Hz}$ ); ${ }^{19} \mathrm{~F}$ NMR $(282 \mathrm{MHz}): \delta-138.7$ (septet, $\mathrm{J}=21.2 \mathrm{~Hz}$ ); LRMS (EI) $\mathrm{mz}$ (relative intensity): $441\left(\mathrm{M}^{+}, 15\right), 151$ (100), 178 (50); HRMS (EI) $\mathrm{m} / z$ calculated for $\mathrm{C}_{21} \mathrm{H}_{28} \mathrm{FNO}_{6} \mathrm{~S}: 441.1621$ found 441.1616.<smiles>COc1ccc(CN(Cc2ccc(OC)cc2OC)S(=O)(=O)C(C)(F)F)c(OC)c1</smiles>

$\mathrm{N}, \mathrm{N}$-Bis(2,4-dimethoxybenzyl)-1,1-difluoro-ethanesulfonamide (53): Sulfonamide $\mathbf{5 0}$ (93.3 mg, $0.218 \mathrm{mmole}),[1.15 \mathrm{M}] n$-BuLi (250 $\mu \mathrm{L}, 0.284 \mathrm{mmole})$, HMPA (190 $\mu \mathrm{L}, 1.09$ mmole) in THF ( $6 \mathrm{~mL})$, and NSFI (103 $\mathrm{mg}, 0.327 \mathrm{mmole})$ in THF ( $2 \mathrm{~mL}+1 \mathrm{~mL}$ rinse). Flash chromatography (Benzene) yielded $32.1 \mathrm{mg}$ (33\%) as a pale yellow solid. Mp 72$73^{\circ} \mathrm{C} ;{ }^{1} \mathrm{H}$ NMR (300 MHz): $\delta 7.16(2 \mathrm{H}, \mathrm{d}, \mathrm{J}=8.3 \mathrm{~Hz}), 6.39(2 \mathrm{H}, \mathrm{dd}, \mathrm{J}=8.3,1.9 \mathrm{~Hz})$, $6.28(2 \mathrm{H}, \mathrm{d}, \mathrm{J}=1.9 \mathrm{~Hz}), 4.48(4 \mathrm{H}, \mathrm{s}), 3.77(6 \mathrm{H}, \mathrm{s}), 3.64(6 \mathrm{H}, \mathrm{s}), 1.98(3 \mathrm{H}, \mathrm{t}, \mathrm{J}=18.5 \mathrm{~Hz})$; ${ }^{13} \mathrm{C}$ NMR (75 MHz): $\delta 160.3,158.1,130.2,124.5(\mathrm{t}, \mathrm{J}=278.9 \mathrm{~Hz}), 116.5,103.8,97.7$, 55.3, 54.9, 46.4, 19.3 (t, J = 23.7 Hz); ${ }^{19} \mathrm{~F}$ NMR (282 MHz): $\delta$-95.9 (q, J = $\left.18.5 \mathrm{~Hz}\right)$; LRMS (EI) $m / z$ (relative intensity): $445\left(\mathrm{M}^{+}, 21\right), 151$ (100), 178 (82), 121 (29); HRMS (EI) $m / z$ calculated for $\mathrm{C}_{20} \mathrm{H}_{25} \mathrm{~F}_{2} \mathrm{NO}_{6} \mathrm{~S}: 445.137066$ found 445.137762 .<smiles>CCC(F)(F)S(=O)(=O)N(Cc1ccc(OC)cc1OC)Cc1ccc(OC)cc1OC</smiles>

$N, N$-Bis(2,4-dimethoxybenzyl)-1,1-difluoro-propane-1-sulfonamide (54): Sulfonamide 51 (141.9 mg, 0.321 mmole), [1.15 M] $n$-BuLi (420 $\mu \mathrm{L}, 0.482 \mathrm{mmole})$, HMPA ( $560 \mu \mathrm{L}, 3.20 \mathrm{mmole})$ in THF (10 mL), and NSFI (202 mg, $0.642 \mathrm{mmole})$ in THF $(2 \mathrm{~mL}+1 \mathrm{~mL}$ rinse). Flash chromatography (15:85 EtOAc:Hexanes then Benzene) yielded $73.4 \mathrm{mg}(50 \%)$ yield $59 \%$ as a pale yellow oil; ${ }^{1} \mathrm{H}$ NMR $(300 \mathrm{MHz}): \delta 7.17$ $(2 \mathrm{H}, \mathrm{d}, \mathrm{J}=8.4 \mathrm{~Hz}), 6.38(2 \mathrm{H}, \mathrm{dd}, \mathrm{J}=8.4,2.3 \mathrm{~Hz}), 6.27(2 \mathrm{H}, \mathrm{d}, \mathrm{J}=2.3 \mathrm{~Hz}), 4.49(4 \mathrm{H}, \mathrm{s})$, $3.77(6 \mathrm{H}, \mathrm{s}), 3.63(6 \mathrm{H}, \mathrm{s}), 2.30(2 \mathrm{H}, \mathrm{dt}, \mathrm{J}=18.1,7.5 \mathrm{~Hz}), 1.16(3 \mathrm{H}, \mathrm{t}, \mathrm{J}=7.5 \mathrm{~Hz}) ;{ }^{13} \mathrm{C}$ 
NMR (75 MHz): $\delta 160.3,158.1,130.2,125.6(\mathrm{t}, \mathrm{J}=281.3 \mathrm{~Hz}), 116.7,103.9,97.8,55.3$, 54.9, 46.4, $25.8(\mathrm{t}, \mathrm{J}=21.6 \mathrm{~Hz}), 5.5(\mathrm{t}, \mathrm{J}=4.8 \mathrm{~Hz}) ;{ }^{19} \mathrm{~F}$ NMR $(282 \mathrm{MHz}): \delta-104.7$; LRMS (EI) $m z$ (relative intensity): 459 (M $\left.\mathbf{M}^{+}, 19\right), 178$ (100), 151 (92); HRMS (EI) $m / z$ calculated for $\mathrm{C}_{21} \mathrm{H}_{27} \mathrm{~F}_{2} \mathrm{NO}_{6} \mathrm{~S}: 459.152716$ found 459.152043<smiles>COS(=O)(=O)NC(CCl)Cc1ccccc1</smiles>

$N$-[(1S)-1-Benzyl-2-chloroethyl]methanesulfonamide (55): Known compound prepared according to original paper without modification. ${ }^{7}$<smiles>O=S(=O)(NC(CCl)Cc1ccccc1)OCc1ccccc1</smiles>

$N$-[(1S)-Benzyl-2-chloroethyl]-1-phenylmethanesulfonamide (56): To a cooled $0^{\circ} \mathrm{C}$ solution of amino-alcohol (502 mg, $3.32 \mathrm{mmole})$, triethylamine ( $970 \mu \mathrm{L}, 6.97 \mathrm{mmole})$ and THF $(4 \mathrm{~mL})$ was added solid $\alpha$-toluenesulfonyl chloride $(1.32 \mathrm{~g}, 6.97 \mathrm{mmole})$ portionwise. The resulting mixture was gradually warmed to room temperature and stirred overnight. The mixture was filtered and washed with EtOAc, and the filtrate evaporated to dryness. The crude intermediate was suspended in a mixture of $\mathrm{NaCl}$ (291 $\mathrm{mg}, 4.98 \mathrm{mmole})$ and DMF $(8 \mathrm{~mL})$. The mixture was heated to $80^{\circ} \mathrm{C}$ overnight, then cooled. The reaction was quenched with water, extracted with EtOAc:Hexanes (x3), EtOAc (x2), washed with $1 \mathrm{~N} \mathrm{HCl}$, brine, dried over $\mathrm{MgSO}_{4}$, and concentrated to dryness. Flash chromatography (15:85 to 25:75 EtOAc:Hexanes) yielded $785 \mathrm{mg}(73 \%)$ as a clear colorless oil; ${ }^{1} \mathrm{H}$ NMR $(300 \mathrm{MHz}): \delta$ 7.40-7.25 $(8 \mathrm{H}, \mathrm{m}), 7.14-7.11(2 \mathrm{H}, \mathrm{m}), 4.43(1 \mathrm{H}, \mathrm{d}$, $\mathrm{J}=9.3 \mathrm{~Hz}), 4.12(2 \mathrm{H}, \mathrm{s}), 3.68-3.66(1 \mathrm{H}, \mathrm{m}), 3.48(1 \mathrm{H}, \mathrm{dd}, \mathrm{J}=11.3,4.4 \mathrm{~Hz}), 3.39(1 \mathrm{H}$, $\mathrm{dd}, \mathrm{J}=11.3,3.1 \mathrm{~Hz}), 2.85(2 \mathrm{H}, \mathrm{d}, \mathrm{J}=7.2 \mathrm{~Hz}) ;{ }^{13} \mathrm{C}$ NMR $(75 \mathrm{MHz}): \delta 136.5,130.6,129.2$, 128.9, 128.6, 128.6, 127.0, 60.0, 56.0, 47.5, 38.4; LRMS (EI) $\mathrm{m} / \mathrm{z}$ (relative intensity): $323\left(\mathrm{M}^{+}, 0.5\right), 232\left(\mathrm{M}^{+}-\mathrm{Bn}, 30\right), 91$ (100); (CI: $\left.\mathrm{NH}_{3}\right) 341\left(\mathrm{M}^{+}+\mathrm{NH}_{4}, 100\right)$; HRMS (EI) $\mathrm{m} / \mathrm{z}$ calculated for $\mathrm{C}_{16} \mathrm{H}_{18} \mathrm{ClNO}_{2} \mathrm{~S}: 323.0747$ found 323.0748

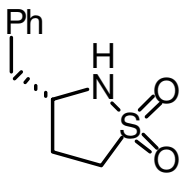


(3S)-3-Benzylisothiazolidine 1,1-dioxide (57): Known compound prepared according to literature without modification.?

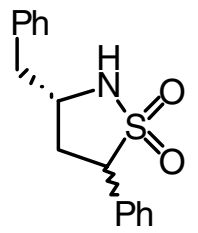

(3S)-3-Benzyl-5-phenylisothiazolidine 1,1-dioxide (58): To a $-70^{\circ} \mathrm{C}$ solution of 56 (630 $\mathrm{mg}, 1.94 \mathrm{mmole}), i \mathrm{Pr}_{2} \mathrm{NH}(68 \mu \mathrm{L}, 0.49 \mathrm{mmole})$, and $\mathrm{THF}(19 \mathrm{~mL})$ was added [1.36 M] $n$ $\mathrm{BuLi}(3.2 \mathrm{~mL}, 4.36 \mathrm{mmole})$ dropwise. Once complete the reaction was gradually warmed to $0^{\circ} \mathrm{C}$ over $1 \mathrm{hr}$. The reaction was stirred at $0^{\circ} \mathrm{C}$ for $1.75 \mathrm{hr}$ then quenched with $1 \mathrm{~N} \mathrm{HCl}$. The product was extracted with EtOAc (x3), washed with brine, dried over $\mathrm{MgSO}_{4}$, filtered and concentrated to dryness. Flash chromatography (25:75 EtOAc:Hexanes) yielded $75.5 \mathrm{mg}$ of the less polar diastereomer and $370.3 \mathrm{mg}$ of a mixtures of the two (80 $\%)$ as a white crystalline solid. M.p. $109.5-110.5^{\circ} \mathrm{C}$; less polar diastereomer: ${ }^{1} \mathrm{H}$ NMR $(300 \mathrm{MHz}): \delta 7.40-7.21(10 \mathrm{H}, \mathrm{m}), 4.48(1 \mathrm{H}, \mathrm{bd}, \mathrm{J}=4.3 \mathrm{~Hz}), 4.37(1 \mathrm{H}, \mathrm{t}, \mathrm{J}=8.7 \mathrm{~Hz})$, 3.97-3.92 $(1 \mathrm{H}, \mathrm{m}), 3.01-2.87(2 \mathrm{H}, \mathrm{m}), 2.84-2.74(1 \mathrm{H}, \mathrm{m}), 2.57-2.48(1 \mathrm{H}, \mathrm{m}) ;{ }^{13} \mathrm{C} \mathrm{NMR}$ (75 MHz): $\delta 136.5,130.8,129.2,129.0,128.9,128.9,127.2,61.5,52.6,42.3,33.7$; more polar isomer: ${ }^{1} \mathrm{H}$ NMR $(300 \mathrm{MHz}): \delta 7.43-7.18(10 \mathrm{H}, \mathrm{m}), 4.79(1 \mathrm{H}, \mathrm{d}, \mathrm{J}=6.4 \mathrm{~Hz}), 4.30$ $(1 \mathrm{H}, \mathrm{dd}, \mathrm{J}=13.1,6.9 \mathrm{~Hz}), 3.82-3.75(1 \mathrm{H}, \mathrm{m}), 2.94(1 \mathrm{H}, \mathrm{dd}, \mathrm{J}=13.5,7.6 \mathrm{~Hz}), 2.81(1 \mathrm{H}$, $\mathrm{dd}, \mathrm{J}=13.5,6.2 \mathrm{~Hz}), 2.57(1 \mathrm{H}$, quintet, $\mathrm{J}=6.6 \mathrm{~Hz}), 2.39(1 \mathrm{H}, \mathrm{dq}, \mathrm{J}=13.1,10.1 \mathrm{~Hz}) ;{ }^{13} \mathrm{C}$ NMR (75 MHz): $\delta$ 137.0, 130.2, 129.1, 129.0, 128.8, 128.7, 128.6, 126.8, 64.7, 53.5, 42.4, 35.1; LRMS (EI) $m / z$ (relative intensity): $287\left(\mathrm{M}^{+}, 5\right), 196\left(\mathrm{M}^{+}-\mathrm{C}_{7} \mathrm{H}_{7}, 60\right), 132$ (100); (CI: $\left.\mathrm{NH}_{3}\right) 305\left(\mathrm{M}^{+}+\mathrm{NH}_{4}, 100\right)$; HRMS (EI) $m / z$ calculated for $\mathrm{C}_{16} \mathrm{H}_{17} \mathrm{NO}_{2} \mathrm{~S}$ : 287.0980 found 287.0974 .

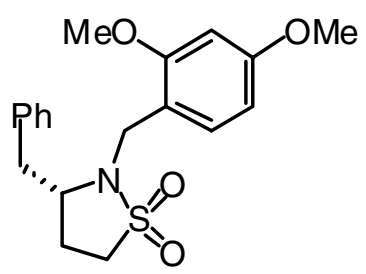

(3S)-3-Benzyl-2-(2,4-dimethoxybenzyl)isothiazolidine 1,1-dioxide (59): To a $0^{\circ} \mathrm{C}$ solution of sultam 57 (120 mg, 0.568 mmole), $\mathrm{PPh}_{3}$ (223 mg, 0.852 mmole), dimethoxybenzyl alcohol (105 mg, $0.625 \mathrm{mmole})$ and THF $(3 \mathrm{~mL})$ was added DEAD (134 $\mu \mathrm{L}, 0.852 \mathrm{mmole})$ dropwise. The reaction was gradually warmed to room temperature and stirred overnight. The solvent was removed in vacou, and the crude mixture purified by flash chromatography (15:85 to 25:75 to 33:67 EtOAc:Hexanes) yielding $123.9 \mathrm{mg}(60 \%)$ as a clear crystalline solid. M.p. $66-67^{\circ} \mathrm{C} ;{ }^{1} \mathrm{H}$ NMR $(300 \mathrm{MHz})$ : $\delta 7.42(1 \mathrm{H}, \mathrm{d}, \mathrm{J}=8.3 \mathrm{~Hz}), 7.30-7.19(3 \mathrm{H}, \mathrm{m}), 7.08-7.05(2 \mathrm{H}, \mathrm{m}), 6.53-6.47(2 \mathrm{H}, \mathrm{m}), 4.48$ 
$(1 \mathrm{H}, \mathrm{d}, \mathrm{J}=15.3 \mathrm{~Hz}), 4.32(1 \mathrm{H}, \mathrm{d}, \mathrm{J}=15.3 \mathrm{~Hz}), 3.84(3 \mathrm{H}, \mathrm{s}), 3.82(3 \mathrm{H}, \mathrm{s}), 3.51-3.44(1 \mathrm{H}$, $\mathrm{m}), 3.15-3.05(2 \mathrm{H}, \mathrm{m}), 2.92-2.82(1 \mathrm{H}, \mathrm{m}), 2.54(1 \mathrm{H}, \mathrm{dd}, \mathrm{J}=13.5,9.8 \mathrm{~Hz}), 2.17-1.98(2 \mathrm{H}$, $\mathrm{m}) ;{ }^{13} \mathrm{C}$ NMR (75 MHz): $\delta$ 160.5, 158.3, 136.6, 131.4, 129.0, 128.4, 126.5, 116.5, 104.4, 98.1, 58.3, 55.2, 55.2, 46.1, 40.8, 39.9, 24.2; LRMS (EI) $m z$ (relative intensity): 361 ( $\mathbf{M}^{+}$, 2), $296\left(\mathrm{M}^{+}-\mathrm{SO}_{2} \mathrm{H}, 12\right), 151$ (100) HRMS (EI) $m / z$ calculated for $\mathrm{C}_{19} \mathrm{H}_{23} \mathrm{NO}_{4} \mathrm{~S}: 361.1348$ found: 361.1352 .

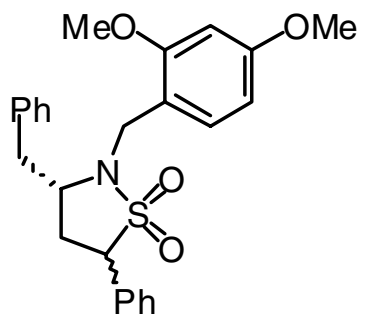

(3S)-3-Benzyl-2-(2,4-dimethoxybenzyl)-5-phenylisothiazolidine 1,1-dioxide (60): To a $0^{\circ} \mathrm{C}$ solution of sultam 58 (120 mg, $\left.0.417 \mathrm{mmole}\right), \mathrm{PPh}_{3}(219 \mathrm{mg}, 0.835$ mmole), dimethoxybenzyl alcohol (140 mg, $0.835 \mathrm{mmole})$ and THF ( $3 \mathrm{~mL})$ was added DIAD (164 $\mu \mathrm{L}, 0.835 \mathrm{mmole})$ dropwise. The reaction was gradually warmed to room temperature and stirred overnight. The solvent was removed in vacou, and the crude mixture purified by flash chromatography (15:85 EtOAc:Hexanes) yielded $113.8 \mathrm{mg}$ $(62 \%)$ as a pale yellow solid. Mp $89-90^{\circ} \mathrm{C}$; less polar isomer ${ }^{1} \mathrm{H}$ NMR (300 MHz): $\delta$ 7.39$7.23(9 \mathrm{H}, \mathrm{m}), 7.12(2 \mathrm{H}, \mathrm{d}, \mathrm{J}=8.0 \mathrm{~Hz}), 6.52-6.48(2 \mathrm{H}, \mathrm{m}), 4.52(1 \mathrm{H}, \mathrm{d}, \mathrm{J}=15.0 \mathrm{~Hz}), 4.40$ $(1 \mathrm{H}, \mathrm{d}, \mathrm{J}=15.0 \mathrm{~Hz}), 4.18(1 \mathrm{H}, \mathrm{dd}, \mathrm{J}=10.7,7.3 \mathrm{~Hz}), 3.86(3 \mathrm{H}, \mathrm{s}), 3.82(3 \mathrm{H}, \mathrm{s}), 3.67-3.61$ $(1 \mathrm{H}, \mathrm{m}), 3.10(1 \mathrm{H}, \mathrm{dd}, \mathrm{J}=13.5,3.6 \mathrm{~Hz}), 2.69(1 \mathrm{H}, \mathrm{dd}, \mathrm{J}=13.5,9.4 \mathrm{~Hz}), 2.51-2.43(1 \mathrm{H}$, $\mathrm{m}), 2.32(1 \mathrm{H}$, ddd, $\mathrm{J}=13.5,7.3,2.9 \mathrm{~Hz}) ;{ }^{13} \mathrm{C}$ NMR $(75 \mathrm{MHz}): \delta 160.8,158.7,136.9$, 132.3, 130.7, 129.4, 128.9, 128.7, 128.6, 126.8, 116.3, 104.6, 98.3, 60.2, 55.4, 55.4, 55.0, 39.8, 39.2, 29.5; more polar isomer ${ }^{1} \mathrm{H}$ NMR $(300 \mathrm{MHz}): \delta 7.49(1 \mathrm{H}, \mathrm{d}, \mathrm{J}=8.3 \mathrm{~Hz})$, 7.44-7.39 (2H, m), 7.37-7.17 (6H, m), 7.10-7.08 $(2 \mathrm{H}, \mathrm{m}), 6.54-6.48(2 \mathrm{H}, \mathrm{m}), 4.58(1 \mathrm{H}, \mathrm{d}$, $\mathrm{J}=15.3 \mathrm{~Hz}), 4.41(1 \mathrm{H}, \mathrm{d}, \mathrm{J}=15.3 \mathrm{~Hz}), 3.93(1 \mathrm{H}, \mathrm{dd}, \mathrm{J}=11.8,8.5 \mathrm{~Hz}), 3.85(3 \mathrm{H}, \mathrm{s}), 3.81$ $(3 \mathrm{H}, \mathrm{s}), 3.49-3.46(1 \mathrm{H}, \mathrm{m}), 3.28(1 \mathrm{H}, \mathrm{dd}, \mathrm{J}=13.1,4.2 \mathrm{~Hz}), 2.60(1 \mathrm{H}, \mathrm{dd}, \mathrm{J}=13.1,10.1$ $\mathrm{Hz}), 2.32-2.25(2 \mathrm{H}, \mathrm{m}) ;{ }^{13} \mathrm{C} \mathrm{NMR}(75 \mathrm{MHz}): \delta 160.6,158.6,136.7,131.7,130.3,129.0$, 128.9, 128.7, 128.5, 126.6, 116.7, 104.6, 98.2, 63.1, 56.8, 55.3, 55.3, 43.1, 41.3, 31.7; LRMS (EI) $m / z$ (relative intensity): $437\left(\mathrm{M}^{+}, 1\right), 346\left(\mathrm{M}^{+}-\mathrm{C}_{7} \mathrm{H}_{7}, 5\right), 151$ (100); HRMS (EI) $m / z$ calculated for $\mathrm{C}_{25} \mathrm{H}_{27} \mathrm{NO}_{4} \mathrm{~S}: 437.1661$ found 437.1675 


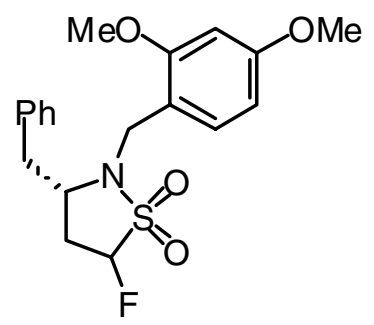

(3S)-3-Benzyl-2-(2,4-dimethoxybenzyl)-5-fluoroisothiazolidine 1,1-dioxide (61): To a cooled $-78^{\circ} \mathrm{C}$ solution of sultam $59(140 \mathrm{mg}, 0.387 \mathrm{mmole}), i \operatorname{Pr}_{2} \mathrm{NH}(76 \mu \mathrm{L}, 0.542$ mmole), HMPA ( $340 \mu \mathrm{L}, 1.93 \mathrm{mmole})$, and THF (12 mL) was added [1.49 M] $n$-BuLi (312 $\mu \mathrm{L}, 0.464$ mmole) slowly. The mixture was stirred at $-78^{\circ} \mathrm{C}$ for $1 \mathrm{hr}$ before a solution of NSFI (183 mg, $0.581 \mathrm{mmole})$ and THF $(2 \mathrm{~mL}+1 \mathrm{~mL}$ rinse) was added via cannula. The reaction was stirred at $-78^{\circ} \mathrm{C}$ for $2 \mathrm{hr}$ then warmed to room temperature and stirred $2 \mathrm{hr}$ further. The reaction was quenched with aq. $\mathrm{NH}_{4} \mathrm{Cl}$, extracted with EtOAc (x3), washed with aq. $\mathrm{NaHCO}_{3}$, brine, dried over $\mathrm{MgSO}_{4}$, filtered and concentrated to dryness. Flash chromatography (15:85 to 25:75 EtOAc:Hexanes) yielded $36.2 \mathrm{mg}$ (25\%) of sultam 61 as a white solid, and $6.6 \mathrm{mg}(4 \%)$ of sultam 62 and $20.2 \mathrm{mg}(14 \%)$ of sultam $\mathbf{6 3}$ while recovering $53.2 \mathrm{mg}(38 \%)$ of sultam 59. $\mathrm{Mp} 74^{\circ} \mathrm{C}$; ${ }^{1} \mathrm{H}$ NMR $(300 \mathrm{MHz}): \delta 7.38$ $(1 \mathrm{H}, \mathrm{d}, \mathrm{J}=8.2 \mathrm{~Hz}), 7.29-7.21(3 \mathrm{H}, \mathrm{m}), 7.04-7.01(2 \mathrm{H}, \mathrm{m}), 6.52-6.47(2 \mathrm{H}, \mathrm{m}), 5.39(1 \mathrm{H}$, dd, J = 52.2, $2.4 \mathrm{~Hz}), 4.63(1 \mathrm{H}, \mathrm{d}, \mathrm{J}=15.0 \mathrm{~Hz}), 4.33(1 \mathrm{H}, \mathrm{d}, \mathrm{J}=15.0 \mathrm{~Hz}), 3.85(3 \mathrm{H}, \mathrm{s})$, $3.82(3 \mathrm{H}, \mathrm{s}), 3.66-3.61(1 \mathrm{H}, \mathrm{m}), 3.14(1 \mathrm{H}, \mathrm{dd}, \mathrm{J}=13.2,4.3 \mathrm{~Hz}), 2.51(1 \mathrm{H}, \mathrm{dd}, \mathrm{J}=13.2$, $10.1 \mathrm{~Hz}), 2.25(1 \mathrm{H}, \mathrm{dd}, \mathrm{J}=7.9,2.4 \mathrm{~Hz}), 2.18-2.11(1 \mathrm{H}, \mathrm{m}) ;{ }^{13} \mathrm{C}$ NMR $(75 \mathrm{MHz}): \delta 160.9$, 158.7, 136.1, 131.7, 129.1, 128.7, 126.9, 116.2, 104.5, 98.3, 94.6 (d, J = 220.4 Hz), 56.4, 55.4, 55.4, 43.4, 41.1, $31.5(\mathrm{~d}, \mathrm{~J}=19.0 \mathrm{~Hz}) ;{ }^{19} \mathrm{~F}$ NMR (282 MHz): $\delta$-176.1; LRMS (EI) $m / z$ (relative intensity): $379\left(\mathrm{M}^{+}, 8\right), 151$ (100); HRMS (EI) $m / z$ calculated for $\mathrm{C}_{19} \mathrm{H}_{22} \mathrm{FNO}_{4} \mathrm{~S}: 379.1284$ found 379.1270

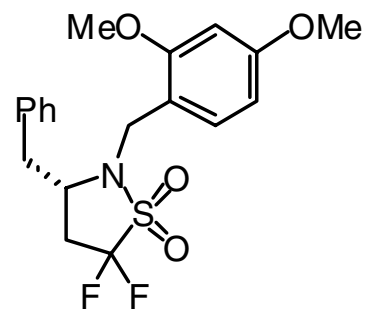

(3S)-3-Benzyl-2-(2,4-dimethoxybenzyl)-5,5-difluoroisothiazolidine 1,1-dioxide (62): See procedure for sultam 61 yielded $6.6 \mathrm{mg}(4 \%)$ as a clear colorless film; ${ }^{1} \mathrm{H}$ NMR (300 $\mathrm{MHz}): \delta$ 7.34-7.23 $(4 \mathrm{H}, \mathrm{m}), 7.02(2 \mathrm{H}, \mathrm{d}, \mathrm{J}=7.8 \mathrm{~Hz}), 6.53-6.48(2 \mathrm{H}, \mathrm{m}), 4.53(1 \mathrm{H}, \mathrm{d}, \mathrm{J}=$ $14.9 \mathrm{~Hz}), 4.39(1 \mathrm{H}, \mathrm{d}, \mathrm{J}=14.9 \mathrm{~Hz}), 3.86(3 \mathrm{H}, \mathrm{s}), 3.83(3 \mathrm{H}, \mathrm{s}), 3.49-3.47(1 \mathrm{H}, \mathrm{m}), 3.24-$ $3.19(1 \mathrm{H}, \mathrm{m}), 2.56(1 \mathrm{H}, \mathrm{dd}, \mathrm{J}=13.1,10.7 \mathrm{~Hz}), 2.39-2.27(2 \mathrm{H}, \mathrm{m}) ;{ }^{13} \mathrm{C}$ NMR $(75 \mathrm{MHz}): \delta$ 161.2, 158.8, 135.6, 132.0, 129.1, 128.9, 127.2, 125.4 (t, J could not be resolved), 115.2, 104.7, 98.5, 55.4, 55.4, $52.3(\mathrm{~d}, \mathrm{~J}=3.9 \mathrm{~Hz}), 42.6,40.1,32.6(\mathrm{t}, \mathrm{J}=19.8 \mathrm{~Hz}) ;{ }^{19} \mathrm{~F}$ NMR (282 MHz): $\delta$-105.7 (d, J = 208.9 Hz), $-109.8(\mathrm{~d}, \mathrm{~J}=208.9 \mathrm{~Hz}$ ); LRMS (EI) $m / z$ (relative 
intensity): $397\left(\mathrm{M}^{+}, 11\right), 151$ (100); HRMS (EI) $\mathrm{m} / \mathrm{z}$ calculated for $\mathrm{C}_{19} \mathrm{H}_{21} \mathrm{~F}_{2} \mathrm{NO}_{4} \mathrm{~S}$ : 397.1159 found 397.1131

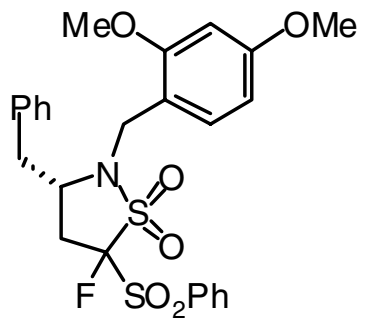

(3S)-3-Benzyl-2-(2,4-dimethoxybenzyl)-5-fluoro-5-(phenylsulfonyl)isothiazolidine 1,1-dioxide (63): See procedure for sultam 61, yielded $20.2 \mathrm{mg}(14 \%)$; ${ }^{1} \mathrm{H}$ NMR (300 $\mathrm{MHz}): \delta 8.06(2 \mathrm{H}, \mathrm{d}, \mathrm{J}=7.9 \mathrm{~Hz}), 7.78(1 \mathrm{H}, \mathrm{t}, \mathrm{J}=7.5 \mathrm{~Hz}), 7.65-7.60(2 \mathrm{H}, \mathrm{m}), 7.31-7.19$ $(4 \mathrm{H}, \mathrm{m}), 7.04(2 \mathrm{H}, \mathrm{d}, \mathrm{J}=6.2 \mathrm{~Hz}), 6.47-6.44(2 \mathrm{H}, \mathrm{m}), 4.51(1 \mathrm{H}, \mathrm{d}, \mathrm{J}=14.6 \mathrm{~Hz}), 4.19(1 \mathrm{H}$, $\mathrm{d}, \mathrm{J}=14.6 \mathrm{~Hz}), 3.82(3 \mathrm{H}, \mathrm{s}), 3.80(3 \mathrm{H}, \mathrm{s}), 3.58-3.53(1 \mathrm{H}, \mathrm{m}), 3.10(1 \mathrm{H}, \mathrm{dd}, \mathrm{J}=13.3,4.4$ $\mathrm{Hz}), 2.87(1 \mathrm{H}, \mathrm{ddd}, \mathrm{J}=38.3,15.3,7.7 \mathrm{~Hz}), 2.62(1 \mathrm{H}, \mathrm{dd}, \mathrm{J}=13.2,10.5 \mathrm{~Hz}), 2.45(1 \mathrm{H}$, ddd, $\mathrm{J}=19.1,15.3,8.0 \mathrm{~Hz}) ;{ }^{13} \mathrm{C}$ NMR $(75 \mathrm{MHz}): \delta 161.1,158.7,135.6,135.5,133.9$, $131.9,130.6,129.2,129.0,128.8,127.1,115.2,110.7(\mathrm{~d}, \mathrm{~J}=261.3 \mathrm{~Hz}), 104.5,98.3$, 55.3, 55.3, 54.3, 45.5, 41.0, $30.7(\mathrm{~d}, \mathrm{~J}=17.7 \mathrm{~Hz}) ;{ }^{19} \mathrm{~F}$ NMR $(282 \mathrm{MHz}): \delta-149.3$ (coupled, dd, J = 38.3, $19.1 \mathrm{~Hz}$ ); LRMS (EI) $m / z$ (relative intensity): $519\left(\mathrm{M}^{+}, 2\right), 151$ (100); (CI: $\left.\mathrm{NH}_{3}\right): 537\left(\mathrm{M}^{+}+\mathrm{NH}_{4}, 100\right), 151$ (55); HRMS (EI) $\mathrm{m} / \mathrm{z}$ calculated for $\mathrm{C}_{25} \mathrm{H}_{26} \mathrm{FNO}_{6} \mathrm{~S}_{2}: 519.1186$ found 519.1188 .

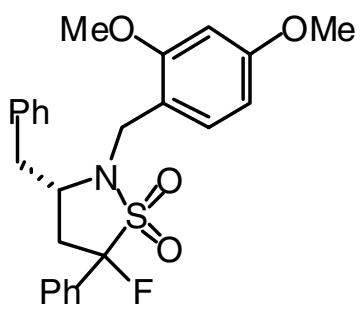

(3S)-3-Benzyl-2-(2,4-dimethoxybenzyl)-5-fluoro-5-phenylisothiazolidine 1,1-dioxide (64): To a stirred $-78^{\circ} \mathrm{C}$ solution of sultam 60 (94 $\left.\mathrm{mg}, 0.215 \mathrm{mmole}\right)$ and THF (6 mL) was added [1.0 M] NaHMDS (430 $\mu \mathrm{L}, 430$ mmole) slowly. The reaction was stirred $1 \mathrm{hr}$ at $-78^{\circ} \mathrm{C}$ then a solution of NSFI (149 $\left.\mathrm{mg}, 0.473 \mathrm{mmole}\right)$ and THF $(2 \mathrm{~mL}+1 \mathrm{~mL}$ rinse) was added via cannula. The reaction was stirred $2 \mathrm{hr}$ at $-78^{\circ} \mathrm{C}$ and $3 \mathrm{hr}$ at room temperature. The reaction was quenched with aq. $\mathrm{NH}_{4} \mathrm{Cl}$, extracted with EtOAc (x3), washed with aq. $\mathrm{NaHCO}_{3}$, brine, dried over $\mathrm{MgSO}_{4}$, filtered and dried. Flash chromatography (15:85 EtOAc:Hexanes) yielded $46.3 \mathrm{mg}$ of the less polar isomer and $42.4 \mathrm{mg}$ of a mixture $(90 \%)$ as an amorphous solid; less polar isomer ${ }^{1} \mathrm{H}$ NMR (300 $\mathrm{MHz}): \delta$ 7.63-7.60 $(2 \mathrm{H}, \mathrm{m}), 7.48-7.42(4 \mathrm{H}, \mathrm{m}), 7.30-7.21(4 \mathrm{H}, \mathrm{m}), 7.08-7.06(2 \mathrm{H}, \mathrm{m})$, 6.56-6.50 (2H, m), $4.75(1 \mathrm{H}, \mathrm{d}, \mathrm{J}=14.9 \mathrm{~Hz}), 4.38(1 \mathrm{H}, \mathrm{d}, \mathrm{J}=14.9 \mathrm{~Hz}), 3.88(3 \mathrm{H}, \mathrm{s}), 3.83$ $(3 \mathrm{H}, \mathrm{s}), 3.72-3.69(1 \mathrm{H}, \mathrm{m}), 3.17(1 \mathrm{H}, \mathrm{dd}, \mathrm{J}=13.3,4.1 \mathrm{~Hz}), 2.67-2.50(2 \mathrm{H}, \mathrm{m}), 2.42(1 \mathrm{H}$, 
dt, J = 15.7, 7.3 Hz) ${ }^{13} \mathrm{C}$ NMR (75 MHz): $\delta 160.9,158.7,136.2,132.0,130.2,130.1(\mathrm{~d}, \mathrm{~J}$ $=21.4 \mathrm{~Hz}), 129.0,128.7,128.6,126.8,126.6(\mathrm{~d}, \mathrm{~J}=3.9 \mathrm{~Hz}), 116.5,104.2(\mathrm{~d}, \mathrm{~J}=215.4$ $\mathrm{Hz}), 98.3,55.9,55.4,55.3,45.4,41.2,35.8(\mathrm{~d}, \mathrm{~J}=19.4 \mathrm{~Hz}) ;{ }^{19} \mathrm{~F}$ NMR $(282 \mathrm{MHz}): \delta-$ 148.8; LRMS (EI) $m / z$ (relative intensity): $455\left(\mathrm{M}^{+}, 5\right), 364\left(\mathrm{M}^{+}-\mathrm{C}_{7} \mathrm{H}_{7}, 2\right), 151$ (100); HRMS (EI) $m / z$ calculated for $\mathrm{C}_{25} \mathrm{H}_{26} \mathrm{FNO}_{4} \mathrm{~S}: 455.1567$ found 455.1563 .

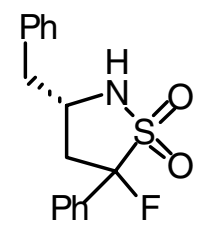

(3S)-3-Benzyl-5-fluoro-5-phenylisothiazolidine 1,1-dioxide (65): TFA (80 $\mu \mathrm{L})$ was added to a cooled $0^{\circ} \mathrm{C}$ solution of sultam $64(11.7 \mathrm{mg}, 0.025 \mathrm{mmole}), \mathrm{CH}_{2} \mathrm{Cl}_{2}(720 \mu \mathrm{L})$ and $i \mathrm{Pr}_{3} \mathrm{SiH}(40 \mu \mathrm{L})$. After $4.5 \mathrm{hr}$ at $0^{\circ} \mathrm{C}$ the solvent was removed in vacou. Flash chromatography (5:95 to 10:90 to 25:75 EtOAc: Hexanes) yielded $5.9 \mathrm{mg}$ (77\%) as a white crystalline solid. M.p. $121.5-122.5^{\circ} \mathrm{C}$; less polar isomer ${ }^{1} \mathrm{H}$ NMR $(300 \mathrm{MHz}): \delta$ 7.66-7.63 (2H, m), 7.50-7.44 (3H, m), 7.42-7.28 $(5 \mathrm{H}, \mathrm{m}), 4.65(1 \mathrm{H}, \mathrm{d}, \mathrm{J}=6.8 \mathrm{~Hz}), 4.05-$ $3.98(1 \mathrm{H}, \mathrm{m}), 3.04(1 \mathrm{H}, \mathrm{dd}, \mathrm{J}=13.5,8.6 \mathrm{~Hz}), 2.94-2.87(2 \mathrm{H}, \mathrm{m}), 2.81-2.76(1 \mathrm{H}, \mathrm{m}) ;{ }^{13} \mathrm{C}$ NMR (75 MHz): $\delta 136.8,130.5,130.3,129.3,128.7(\mathrm{~d}, \mathrm{~J}=3.5 \mathrm{~Hz}), 127.1,126.6(\mathrm{~d}, \mathrm{~J}=$ 7.6 Hz), 121.7, $105.4(\mathrm{~d}, \mathrm{~J}=215.4 \mathrm{~Hz}), 52.0,42.8,38.3(\mathrm{~d}, \mathrm{~J}=19.4 \mathrm{~Hz}) ;{ }^{19} \mathrm{~F}$ NMR $(282$ MHz): $\delta$-147.3; LRMS (EI) $m z$ (relative intensity): $241\left(\mathrm{M}^{+}-\mathrm{SO}_{2}, 12\right), 150$ (100); HRMS $m / z$ calculated for $\mathrm{C}_{16} \mathrm{H}_{16} \mathrm{NF}\left(\mathrm{M}^{+}-\mathrm{SO}_{2}\right): 241.1267$ found 241.1263.

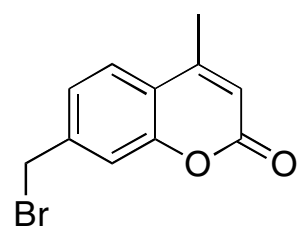

7-Bromomethyl-4-methylcoumarin $(\mathbf{6 6})^{8}$ : To a stirred suspension of 4,7-dimethyl coumarin ${ }^{9}(8.70 \mathrm{~g}, 50 \mathrm{mmol})$ in $100 \mathrm{~mL}$ of dry benzene was NBS $(9.35 \mathrm{~g}, 52.5 \mathrm{mmols})$ and benzoyl preoxide $(115 \mathrm{mg}, 0.5 \mathrm{mmol})$. The resulting mixture was heated to reflux overnight $(16 \mathrm{~h})$, cooled to room temperature, and filtered. Concentration of the filtrate in vacuo provided a pale yellow solid, which was washed thoroughly with water. The solids was recrystallized from ethanol to give $11.29 \mathrm{~g}$ of (89\%) 66 as a white crystal. ${ }^{1} \mathrm{H}$ NMR $\left(\mathrm{CDCl}_{3}\right) 7.56(\mathrm{~d}, 1 \mathrm{H}, J=8.0 \mathrm{~Hz}), 7.32(\mathrm{~s}, 1 \mathrm{H}), 7.31(\mathrm{~d}, 1 \mathrm{H}, J=8.3 \mathrm{~Hz}), 6.28(\mathrm{~s}, 1 \mathrm{H})$, $4.50(\mathrm{~s}, 2 \mathrm{H}), 2.41(\mathrm{~s}, 3 \mathrm{H})$.

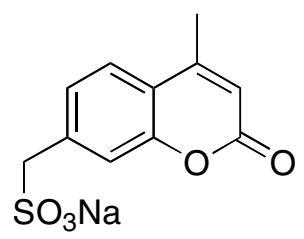


Sodium 7-(4-methylcoumarin) methane sulfonate (67): To a suspension of 66 (6.075 $\mathrm{g}, 24 \mathrm{mmol})$ in $135 \mathrm{~mL}$ of ethanol was added a solution of $\mathrm{Na}_{2} \mathrm{SO}_{3}(3.600 \mathrm{~g}, 28.6 \mathrm{mmol})$ in $\mathrm{H}_{2} \mathrm{O}(135 \mathrm{~mL})$. The resulting mixture was refluxed $5 \mathrm{~h}$, cooled to room temperature. After removal of the solvents, the residue was recrystallized from water to afford $5.05 \mathrm{~g}$ $(76 \%)$ of 67 as a colorless crystal. ${ }^{1} \mathrm{H}$ NMR (DMSO- $\left.d_{6}\right) 7.56(\mathrm{~s}, 1 \mathrm{H}), 7.24(\mathrm{~s}, 2 \mathrm{H}), 6.24$ (s, 1H), 3.89 (s, 2H), 2.32 (s, 3H); ${ }^{13} \mathrm{C}$ NMR (DMSO- $\left.d_{6}, 75 \mathrm{MHz}\right) 160.6,153.8,152.9$, 140.2, 127.2, 125.0, 118.4, 118.2, 114.0, 57.4, 18.6; HRMS (ESI) calcd for $\mathrm{C}_{11} \mathrm{H}_{9} \mathrm{O}_{5} \mathrm{~S}$ 253.0176, found 253.0188.

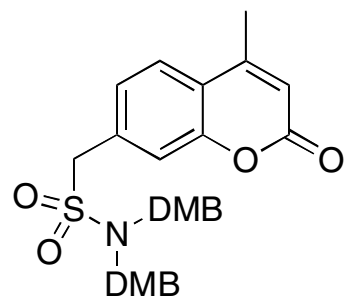

$\mathrm{N}, \mathrm{N}$-Bis(2,4-dimethoxylbenzyl) 7-(4-methylcoumarin) methane sulfonamide (68): To a suspension of $67(3.00 \mathrm{~g}, 10.9 \mathrm{mmol})$ in $120 \mathrm{~mL}$ of dry benzene was added DMF $(0.40$ $\mathrm{mL}$ ) and thionyl chloride $\left(5 \mathrm{~mL}\right.$ ). The resulting mixture was heated at $86^{\circ} \mathrm{C}$ (oil bath temperature) for $5 \mathrm{~h}$., cooled to room temperature. After removal of the solvents in vacuo, the residue was washed with ice cold water $(50 \mathrm{~mL})$ and then transferred to a $250 \mathrm{~mL}$ of round bottom flask. Acetone was added to remove the water and the flask was dried under vacuum line for $1 \mathrm{~h}$. DMAP $(1.20 \mathrm{~g}, 10 \mathrm{mmol}), 6$ (3.00 g, $9.5 \mathrm{mmol})$ and THF (100 $\mathrm{mL}$ ) were added. The resulting mixture was heated to reflux overnight, cooled to room temperature. After removal of the solvents, the residue was redissolved in $200 \mathrm{~mL}$ of $\mathrm{CH}_{2} \mathrm{Cl}_{2}$, washed successively with water $(50 \mathrm{~mL}), 10 \%$ citric acid $(50 \mathrm{mLx} 2)$, water (50 $\mathrm{mL})$ and brine $(50 \mathrm{~mL})$, dried over $\mathrm{Na}_{2} \mathrm{SO}_{4}$. Purification by flash column chromatography (1:1) EtOAc/hexane gave $3.70 \mathrm{~g}(62 \%$ over two steps from 67$)$ of $\mathbf{6 8}$ as a white solid. ${ }^{1} \mathrm{H}$ NMR $\left(\mathrm{CDCl}_{3}\right) 7.47(\mathrm{~d}, 1 \mathrm{H}, J=8.1 \mathrm{~Hz}), 7.27-7.17(\mathrm{~m}, 3 \mathrm{H}), 6.88(\mathrm{~s}, 1 \mathrm{H}), 6.43-6.40(\mathrm{~m}$, $4 \mathrm{H}), 6.23(\mathrm{~s}, 1 \mathrm{H}), 4.20(\mathrm{~s}, 4 \mathrm{H}), 4.06(\mathrm{~s}, 2 \mathrm{H}), 3.77(\mathrm{~s}, 6 \mathrm{H}), 3.77(\mathrm{~s}, 6 \mathrm{H}), 2.36(\mathrm{~s}, 3 \mathrm{H}) ;{ }^{13} \mathrm{C}$ NMR $\left(\mathrm{CDCl}_{3}, 75 \mathrm{MHz}\right) 160.8,160.7,158.5,153.2,152.3,133.9,131.5,126.8,124.7$, $119.8,119.2,117.0,115.3,104.3,98.4,58.8,55.5,55.3,45.9,18.7$; LRMS (EI), $\mathrm{m} / \mathrm{z}$ (relative intensity), $553\left(\mathrm{M}^{+}, 20\right), 338$ (10), 316 (25), 178 (75), 151 (100), 121 (34); HRMS (EI) calcd for $\mathrm{C}_{29} \mathrm{H}_{31} \mathrm{NO}_{8} \mathrm{~S} 553.1770$, found 553.1769.

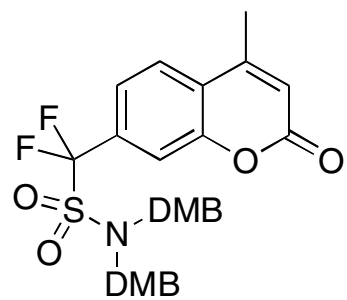

$\mathrm{N}, \mathrm{N}$-Bis(2,4-dimethoxylbenzyl) 7-(4-methylcoumarin) difluoromethane sulfonamide (69): To a solution of $68(553 \mathrm{mg}, 1 \mathrm{mmol})$ and NFSi $(788 \mathrm{mg}, 2.5 \mathrm{mmol})$ in $60 \mathrm{~mL}$ of THF at $-78{ }^{\circ} \mathrm{C}$ was added NaHMDS (1.0 M in THF, $\left.2.5 \mathrm{~mL}, 2.5 \mathrm{mmol}\right)$ via a syringe pump over $50 \mathrm{~min}$. After stirring $2.75 \mathrm{~h}$ at that temperature, the reaction was quenched 
with $5 \mathrm{~mL}$ of saturated aq. $\mathrm{NH}_{4} \mathrm{Cl}$ at $-78{ }^{\circ} \mathrm{C} .60 \mathrm{~mL}$ of EtOAc and $20 \mathrm{~mL}$ of $\mathrm{H}_{2} \mathrm{O}$ were added. After separation and extraction with EtOAc $(60 \mathrm{~mL})$, the extracts were combined and washed with $\mathrm{H}_{2} \mathrm{O}(30 \mathrm{~mL})$ and brine $(30 \mathrm{~mL})$, dried over $\mathrm{Na}_{2} \mathrm{SO}_{4}$ The residue was subjected to flash column chromatography (1:2.5 to $1: 1 \mathrm{EtOAc} / \mathrm{hexane})$ to give $311 \mathrm{mg}$ $(53 \%)$ of 69 as white foam. ${ }^{1} \mathrm{H}$ NMR $\left(\mathrm{CDCl}_{3}\right) 7.60(\mathrm{~d}, 1 \mathrm{H}, J=8.1 \mathrm{~Hz}), 7.52(\mathrm{~d}, 1 \mathrm{H}$, overlapping), $7.50(\mathrm{~s}, 1 \mathrm{H}), 7.10(\mathrm{~d}, 2 \mathrm{H}, J=8.4 \mathrm{~Hz}), 6.31(\mathrm{dd}, 2 \mathrm{H}, J=8.4 \mathrm{~Hz}, 1.8 \mathrm{~Hz})$, $6.28(\mathrm{~s}, 1 \mathrm{H}), 6.24(\mathrm{~s}, 2 \mathrm{H}), 4.46(\mathrm{~s}, 4 \mathrm{H}), 3.70(\mathrm{~s}, 6 \mathrm{H}), 3.61(\mathrm{~s}, 6 \mathrm{H}), 2.35(\mathrm{~s}, 3 \mathrm{H}) ;{ }^{13} \mathrm{C} \mathrm{NMR}$ $\left(\mathrm{CDCl}_{3}, 75 \mathrm{MHz}\right) 160.5,159.8,158.2,152.9,151.5,132.9(\mathrm{t}, J=22 \mathrm{~Hz}), 130.5,125.0$, $122.8(\mathrm{t}, J=5 \mathrm{~Hz}), 122.4,121.4(\mathrm{t}, J=282 \mathrm{~Hz}), 116.9,116.4,116.1(\mathrm{t}, J=6 \mathrm{~Hz}), 104.0$, 97.8, 55.3, 55.0, 46.9, 18.6; ${ }^{19} \mathrm{~F}$ NMR $\left(\mathrm{CDCl}_{3}, 282 \mathrm{MHz}\right)-100.9$; LRMS (EI), $\mathrm{m} / \mathrm{z}$ (relative intensity), $589\left(\mathrm{M}^{+}, 38\right), 316$ (12), 209 (40), 178 (72), 151 (100), 121 (30); HRMS (EI) calcd for $\mathrm{C}_{29} \mathrm{H}_{29} \mathrm{~F}_{2} \mathrm{NO}_{8} \mathrm{~S} 589.1582$, found 589.1591.

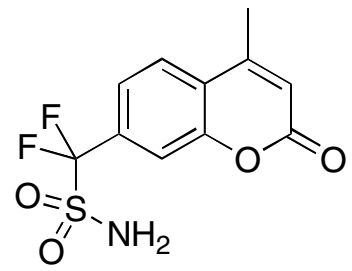

7-(4-methylcoumarin) difluoromethane sulfonamide (2): To a solution of 69 (311 mg, $0.53 \mathrm{mmol}$ ) in $12.5 \mathrm{~mL}$ of $\mathrm{CH}_{2} \mathrm{Cl}_{2}$ at $\mathrm{rt}$ was added $5 \mathrm{~mL}$ of TFA over $10 \mathrm{~min}$. After stirring $2 \mathrm{~h}$ at $\mathrm{rt}$, the solvents were removed in vacuo to give a pink residue. $40 \mathrm{~mL}$ of acetone was added, the mixture was stirred for $15 \mathrm{~min}$. After filtration and rinsing with acetone, the filtrate was subjected to flash column chromatography (1:4 to $1: 2$ acetone/hexane) to afford $105 \mathrm{mg}(69 \%)$ of 2 as a pale white solid. ${ }^{1} \mathrm{H}$ NMR (acetone- $d_{6}$ ) $7.94(\mathrm{~d}, 1 \mathrm{H}, J=8.3 \mathrm{~Hz}), 7.61(\mathrm{~d}, 1 \mathrm{H}, J=8.1 \mathrm{~Hz}), 7.55(\mathrm{~s}, 1 \mathrm{H}), 7.38\left(\mathrm{~s}, 2 \mathrm{H}, \mathrm{NH}_{2}\right), 6.44$ $(\mathrm{s}, 1 \mathrm{H}), 2.52(\mathrm{~s}, 3 \mathrm{H}) ;{ }^{13} \mathrm{C}$ NMR (acetone- $\left.d_{6}, 75 \mathrm{MHz}\right) 158.9,153.0,151.9,132.2(\mathrm{t}, J=23$ $\mathrm{Hz}), 125.7,122.6(\mathrm{t}, J=6 \mathrm{~Hz}), 119.8(\mathrm{t}, J=279 \mathrm{~Hz}), 116.6,115.6(\mathrm{t}, J=6 \mathrm{~Hz}), 17.6 ;{ }^{19} \mathrm{~F}$ NMR (acetone- $\left.d_{6}, 282 \mathrm{MHz}\right)$-103.9; LRMS (EI), $\mathrm{m} / z$ (relative intensity), $289\left(\mathrm{M}^{+}, 6\right)$, 209 (100), 181 (12); HRMS (EI) calcd for $\mathrm{C}_{11} \mathrm{H}_{9} \mathrm{~F}_{2} \mathrm{NO}_{4} \mathrm{~S} 289.0220$, found 289.0210.

\section{REFERENCES}

1. Sulfonyl chlorides were prepared from corresponding sulfonate sodium salts (prepared by reactioning the corresponding benzylic bromide with sodium sulfite) using procedures adapted from literature methods. See: Abdellaoui, H.; Depreux, P.; Lesieur, D.; Pfeiffer, B.; Bontempelli, P. Synthetic. Commun. 1995, 1303 and Sprague, J.M.; Johnson, T.B. J. Am. Chem. Soc. 1937, 59, 1837.

2. N,N-phenyl-2,4-dimethoxybenzyl amine was prepared from reductive amination of aniline and 2,4-dimethoxybenzyl aldehyde.

3. Kaiser, E.M.; Solter, L.E.; Schwarz, R.A.; Beard, R.D.; Hauser, C.R. J. Am. Chem. Soc. 1971, 93, 4237.

4. Baxter, N.J.; Rigoreau, L.J.M.; Laws, A.P.; Page, M.I. J. Am. Chem. Soc. 2000, 122, 3375.

5. Orazi, O.O.; Corral, R.A.; Bravo, R. J. Heterocycl. Chem. 1986, 1701. 
6. Rooney, C.S.; Cochran, D.W.; Ziegler, C.; Cragoe, E.J.; Williams, H.W.R. J. Org. Chem. 1984, 49, 2212.

7. Lee, J.; Zhong, Y-L.; Reamer, R.A.; Askin, D. Org. Lett. 2003, 5, 4175.

8. Shah, D.O.; Trivedi, K.N. Indian J. Chem. 1974, 121, 1014.

9. Osborne, A.G. Tetrahedron, 1981, 37, 2021. 